\title{
Gravity Waves due to a Point Disturbance in a Plane Free Surface Flow of Stratified Fluids
}

\author{
C. C. Mei and T. YaO-Tsu Wu \\ Karmán Laboratory of Fluid Mechanics and Jet Propulsion \\ California Institute of Technology, Pasadena, California \\ (Received 20 April 1964; final manuscript received 8 June 1964)
}

\begin{abstract}
The fundamental solution of the gravity waves due to a two-dimensional point singularity submerged in a steady free-surface flow of a stratified fluid is investigated. A linearized theory is formulated by using Love's equations. The effect of density stratification $\rho_{0}(y)$ and the gravity effect are characterized by two flow parameters $\sigma=-\left(d \rho_{0} / d y\right) / \rho_{0}$ and $\lambda=g L / U^{2}$, where $\lambda^{-1}$ may be regarded as the internal Froude number if $L$ assumes a characteristic value of $\sigma^{-1}$. Two special cases of $\sigma$ and $\lambda$ are treated in this paper. In the first case of constant $\sigma$ (and arbitrary $\lambda$ ) an exact mathematical analysis is carried out. It is shown that the flow is subcritical or supercritical according as $\lambda>$ or $<1 / 2$, in analogy to the corresponding states of channel flows. In addition to a potential surface wave, which exists only for $\lambda>1 / 2$, there arises an internal wave which is attenuated at large distances for $\lambda>1 / 4$ and decays exponentially for $\lambda<1 / 4$. In the second example an asymptotic theory for large $\lambda$ is developed while $\sigma(y)$ may assume the profile roughly resembling the actual situation in an ocean where a pronounced maximum called a seasonal thermocline occurs. Internal waves are now propagated to the downstream infinity in a manner analogous to the channel propagation of sound in an inhomogeneous medium.
\end{abstract}

\section{INTRODUCTION}

$\mathbf{I}^{\mathrm{T}}$ $T$ is a well-known phenomenon that when a layer of lighter fluid is superposed above a heavier one, gravity waves may occur not only at the upper free surface but also at the interface. In an ocean, the occurrence of a top layer of fresh water, usually due to melting of ice, has been attributed to the so-called dead-water phenomenon occasionally encountered by ships. Below a certain critical speed the resulting interfacial wave may become very significant (especially for small density differences) as compared with the free-surface wave. ${ }^{1}$ The interfacial wave so generated is maintained at the expense of an increase in wave resistance on the ship. In the case of several fluids of different density flowing in a channel of finite depth, internal hydraulic jumps have been observed in the supercritical state, as shown by Long. ${ }^{2}$ Various kinds of internal-gravity waves also arise in the atmosphere for which the density gradient of the air is largely responsible. A striking example is the forced oscillation of air passing over a mountain ridge, producing the lee waves, which are sometimes revealed by the appearance of lenticular clouds and motherof-pearl clouds. A discussion of this meteorological problem has been given by Eliassen and Kleinschmidt. ${ }^{3}$

${ }^{1}$ H. Lamb, Hydrodynamics, (Cambridge University Press, London, 1932), 6th ed.

2 R. R. Long, Tellus V, 42 (1953); VI, 97 (1954)

3 A. Eliassen and E. Kleinschmidt, in Handbuch der Physik, edited by $\mathrm{S}$. Flügge (J. Springer-Verlag, Berlin, 1957), Vol. 48, pp. 1-154.
The flow parameter characterizing a continuous density stratification in fluids may often be related to the so-called Väisälä frequency

$$
N^{2}(y)=-g\left(\frac{1}{\rho_{0}} \frac{d \rho_{0}}{d y}+\frac{g}{c^{2}}\right)
$$

where $g$ is the gravitational acceleration, $\rho_{0}$ the static-density distribution of the fluid, and $c$ the local speed of sound. Actual observations in ocean show that $N^{2}$ is usually small in a top layer, rises sharply to a peak at the depth of about $30 \mathrm{~m}$, then gradually decreases, and rises again to a new, smaller second peak at a few hundred meters below, and finally diminishes to zero at great depth. The first and the second peaks are called, respectively, the seasonal and permanent thermoclines. A typical profile, as given by Eckart," is shown in Fig. 1. The magnitude of the second term, $(g / c)^{2}$, is known to be almost a constant (about $0.5 \times 10^{-4} / \mathrm{sec}^{2}$ ) and is generally insignificant compared with the first term $\left(g / \rho_{0}\right)\left(d \rho_{0} / d y\right)$ except at great depths where the first term also becomes small.

For gravity waves in a continuously stratified fluid there exist considerable literatures dealing with the dispersion relations of simple harmonic waves of small amplitude. Yih, ${ }^{5}$ Eckart, $^{6}$ and Tolstoy ${ }^{7}$ have investigated this problem together with the compressibility effect. For the flow motions in an ocean where the compressibility effect is insignificant,

${ }^{4}$ C. Eckart, Phys. Fluids 4, 791 (1961).

${ }^{5}$ C. S. Yih, J. Fluid Mech. 8, 481 (1960).

${ }^{\circ} \mathrm{C}$. Eckart, Hydrodynamics of Oceans and Atmospheres (Pergamon Press, Inc., New York, 1960).

7 I. Tolstoy, Rev. Mod. Phys. 35, 207 (1963). 


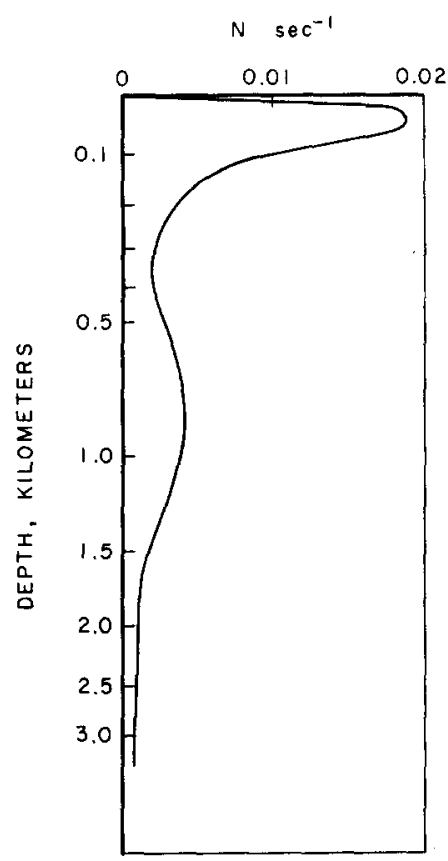

FIG. 1. A typical variation of stability frequency in ocean.

use of the incompressible Väisälä frequency

$$
N^{2}(y)=-\frac{1}{\rho_{0}} \frac{d \rho_{0}}{d y},
$$

is sufficient to bring out the essential features of the internal-gravity waves; this affords the model adopted by Love. ${ }^{8}$ Based on Love's equations. Yanowitch $^{9}$ has investigated the dispersion relations for a fluid with a piece-wise smooth-density variation.

In this paper, attempts are made to generalize the classical dead-water problem to the case of fluids with arbitrary density stratification. A linearized theory of the two-dimensional motion is formulated by using Love's equations and linearized boundary conditions, the flow being assumed to be incompressible. The fundamental solution due to a point singularity (a source or a doublet) submerged beneath the free water surface is derived. In this problem the stratification effect and gravity effect are characterized by two flow parameters

$$
\sigma(y)=-\frac{1}{\rho_{0}} \frac{d \rho_{0}}{d y}, \quad \lambda=\frac{g L}{U^{2}},
$$

where $L$ and $U$ denote a characteristic length and velocity $[L$ may be reckoned as a characteristic value of $\left.\rho_{0} /\left(d \rho_{0} / d y\right)\right]$. The following two specific cases have been treated in detail.

${ }^{8}$ A. E. H. Love, Proc. London Math. Soc. 22, 307 (1891).

${ }^{9} \mathrm{M}$. Yanowitch, Comm. Pure Appl. Math. 15, 45 (1962).
The first is the special case of constant $\sigma(\sigma=1)$, corresponding to an exponential growth of $\rho_{0}$ with depth. With this assumption the problem becomes tractable from a mathematical viewpoint. It is found that an irrotational surface wave exists, only in the lee side, for $\lambda>\frac{1}{2}$ and disappears for $\lambda<\frac{1}{2}$, a result which is quite in analogy to the subcritical and supercritical states in open channel flows of homogeneous fluids. In addition to this wave mode, we find that there is indeed also an internal wave motion, which does not consist of one single harmonic wave as in the two-layered flow case, but is given as an integral of wave elements. The various waves composing this Fourier integral interact, eventually cancelling each other at large distances from the disturbance. This result is primarily due to the simple assumption of uniform $\sigma$. The wave resistance in this case exhibits an interesting feature that it possesses a maximum in the region $\lambda>\frac{1}{2}$ for each fixed submergence depth $h$, and the locus of these maxima has a minimum at $h=2 L$.

In the second case, $\sigma(y)$ is assumed to decrease monotonically with decreasing $y$ (or increasing depth) so as to resemble, to a degree, the shape of a thermocline. An asymptotic theory for large $\lambda$ (or small internal Froude number) is developed here by using Liouville-Langer's method in the theory of differential equations with a large parameter. The internal waves now appear in a number of discrete modes, each confined inside a horizontal channel, propagating to the downstream infinity in a manner similar to the channeled propagation of highfrequency sound waves in an atmosphere with a temperature inversion.

\section{LINEARIZED FORMULATION}

We consider the steady disturbance due to a point source submerged beneath the free surface of a uniform plane flow. The fluid is assumed to be inviscid, incompressible, but stratified. If unperturbed, the free surface coincides with the horizontal plane $y=0$, and the stratification is specified by a given density function

$$
\rho_{0}=\rho_{0}(y), \quad y<0,
$$

the positive $y$ axis pointing vertically upward so that the gravity acts in the negative $y$ direction. The free-stream velocity $U$ is in the positive $x$ direction and is assumed constant. We further assume that both $\rho_{0}$ and $\left(-d \rho_{0} / d y\right)$ are positive, the latter condition being required by the consideration of stability.

It may be remarked here that in a strict sense, 
the fluid issued from the source, whether or not of the same density as that of the fluid passing by the source, will change the basic stratification in the downstream. In practical applications, however, this difficulty does not arise when the net source strength is zero, such as when a solid body is represented by a source distribution, or when a doublet flow is deduced from this fundamental solution.

Thus, with a point source of strength $Q$ submerged at $(0,-h)$, the continuity equation becomes

$$
\left(u \rho_{x}+v \rho_{y}\right) / \rho+u_{x}+v_{y}=Q \delta(x) \delta(y+h),
$$

where $u, v$, are the $x$ - and $y$-component of the velocity, subscripts $x, y$ denote partial differentiations and $\delta(x)$ represents the Dirac function. The momentum equations are

$$
\begin{aligned}
\rho\left(u u_{x}+v u_{y}\right)+p_{x} & =0, \\
\rho\left(u v_{x}+v v_{y}\right)+p_{y}+g \rho & =0,
\end{aligned}
$$

where $p$ is the pressure and $g$ the gravitational constant.

For an incompressible fluid, we assume the following "equation of state"

$$
d \rho / d t=u \rho_{x}+v \rho_{y}=0,
$$

which asserts the invariance of density along a stream line in a steady motion. Now, by (8), (5) is reduced to

$$
u_{x}+v_{y}=Q \delta(x) \delta(y+h) .
$$

This implies that a stream function $\psi$ exists, except at $(0,-h)$, such that

$$
u=\psi_{y}, \quad v=-\psi_{x} .
$$

The momentum equations (6) and (7) can be integrated once under condition (8), giving the Bernoulli equation

$$
\frac{1}{2} q^{2}+\frac{p}{\rho}+g y=H(\psi),
$$

where $q^{2}=u^{2}+v^{2}$, and $H(\psi)$ is a function of $\psi$ to be determined by the conditions far upstream. Thus, if $y_{0}(\psi)$ is the height of the streamline $\psi=$ const as $x \rightarrow \infty$, then

$$
\frac{1}{2} q^{2}(x, y)+\frac{p}{\rho}+g y=\frac{1}{2} U^{2}+\frac{1}{\rho_{0}\left(y_{0}\right)} p_{0}\left(y_{0}\right)+g y_{0},
$$

where

$$
p_{0}(y)=g \int_{y}^{0} \rho_{0}(y) d y
$$

Let the free surface of the flow, not known in advance, be denoted by

$$
F(x, y) \equiv y-\eta_{0}(x)=0 .
$$

As usual, the boundary conditions on the free surface are

$d F / d t=u F_{x}+v F_{y}=0 \quad$ on $\quad F(x, y)=0$,

$d p / d t=u p_{x}+v p_{y}=0$ on $F(x, y)=0$.

Here, the first condition states that kinematically the fluid particles on the free surface will remain on it, and the second condition specifies a constant surface pressure, which may be taken to be zero. Thus, we note from the above set of governing equations and boundary conditions that the problem is intrinsically nonlinear. It may be linearized, however, for small disturbances.

We introduce the perturbation quantities

$$
\begin{gathered}
u=U+u_{1}, \quad v=v_{1}, \\
\rho=\rho_{0}(y)+\rho_{1}, \quad p=p_{0}(y)+p_{1},
\end{gathered}
$$

and assume infinitesimal disturbances so that squares and products of $u_{1}, v, p_{1}, \rho_{1}$ and their derivatives are to be neglected. Thus, Eqs. (6)-(9) are linearized to the following equations, to be valid for $y<0$,

$$
\begin{gathered}
\frac{\partial u_{1}}{\partial x}+\frac{\partial v}{\partial y}=Q \delta(x) \delta(y+h), \\
\rho_{0} U \frac{\partial u_{1}}{\partial x}+\frac{\partial p_{1}}{\partial x}=0, \\
\rho_{0} U \frac{\partial v}{\partial x}+\frac{\partial p_{1}}{\partial y}+g \rho_{1}=0, \\
U\left(\partial \rho_{1} / \partial x\right)+v \rho_{0}^{\prime}(y)=0,
\end{gathered}
$$

where the prime signifies differentiation with respect to $y$. The boundary conditions (14), (15) on the free surface reduce to

$$
\begin{array}{r}
v=U \frac{d \eta_{0}}{d x} \quad \text { on } \quad y=0 \\
U \frac{\partial p_{1}}{\partial x}+v p_{0}^{\prime}(y)=0 \quad \text { on } \quad y=0 .
\end{array}
$$

Furthermore, the perturbation is required to diminish at far upstream and at great depth below the free surface,

$$
\begin{aligned}
& u_{1}, v, \rho_{1}, p_{1} \rightarrow 0 \quad \text { as } \quad x \rightarrow-\infty, \\
& u_{1}, v, \rho_{1}, p_{1} \rightarrow 0 \text { as } y \rightarrow-\infty .
\end{aligned}
$$

In case of a channel of finite depth, the last condition 
is of course replaced by the usual inviscid boundary condition.

Upon elimination of $u_{1}, p_{1}, \rho_{1}$ from (17)-(20), [first eliminating $p_{1}$ by cross differentiation of (18) and (19) and then $u_{1}, \rho_{1}$ by substitution], we obtain a single equation for the vertical velocity component $v$

$$
\begin{aligned}
\rho_{0} U \nabla^{2} v+\rho_{0}^{\prime} U \frac{\partial v}{\partial y}-\frac{g}{U} \rho_{0}^{\prime} v \\
\quad=Q U \rho_{0}(-h) \delta(x) \delta^{\prime}(y+h),
\end{aligned}
$$

where on the right-hand side use has been made of the general theorem

$$
f(y) \delta^{\prime}(y)=f(0) \delta^{\prime}(y)-f^{\prime}(0) \delta(y) .
$$

The corresponding boundary condition of $v$ on the free surface can be obtained by making use of (12), (17), and (18) in (22), giving

$$
(\partial v / \partial y)-\left(g / U^{2}\right) v=0 \text { on } y=0 .
$$

Let $L$ denote a characteristic length of the problem, which can be appropriately chosen in each specific case, so that we can introduce the dimensionless coordinates

$$
\tilde{x}=x / L, \quad \tilde{y}=y / L, \quad \text { and } \quad \tilde{h}=h / L .
$$

We, however, omit in the following the tilde over $x$ and $y$ for brevity, since their being dimensionless or not is rather obvious. Dividing (25) by $\rho_{0} U$, and using again (26), we obtain

$$
\begin{aligned}
\nabla^{2} v & -\sigma(y)\left(\frac{\partial v}{\partial y}-\lambda v\right) \\
& =\frac{Q}{L} \delta(x)\left[\delta^{\prime}(y+h)-\sigma(-h) \delta(y+h)\right]
\end{aligned}
$$

where

$$
\sigma(y)=-\rho_{0}^{\prime}(y) / \rho_{0}(y)>0, \quad \lambda=g L / U^{2} .
$$

Similarly, (27) becomes

$$
\partial v / \partial y-\lambda v=0 \text { on } y=0 .
$$

Thus we see that $\sigma$ and $\lambda$ are two dimensionless flow parameters of this problem; together they characterize the effects of density stratification and gravity.

For the solution of (29) under conditions (31), (23), (24), it suffices to solve for the following associated function $G(x, y)$ defined by

$$
\begin{gathered}
\nabla^{2} G-\sigma\left(G_{y}-\lambda G\right)=\delta(x) \delta(y+h) \text { for } y<0, \\
G_{y}-\lambda G=0 \text { on } y=0
\end{gathered}
$$

and the condition that $G$ vanishes at $x=-\infty$ and $y=-\infty$. Clearly, $v$ and $G$ are related by

$$
v(x, y)=\frac{Q}{L}\left[\frac{\partial}{\partial h}-\sigma(-h)\right] G(x, y ; h) .
$$

When $v$ is solved in this way, $u_{1}$ can be obtained by quadrature from (17), and $p_{1}$ from (18), $\rho_{1}$ from (20).

We next represent $G$ as the real part $(\mathrm{Re})$ of a Fourier integral with the spectrum $f(y, k)$

$$
G(x, y)=\frac{1}{2 \pi} \operatorname{Re} \int_{-\infty}^{\infty} \exp (i k x) f(y, k) d k .
$$

Then, by substitution in (32), (33), assuming no contribution from the integration limits at $x= \pm \infty$, we obtain

$$
\begin{gathered}
f^{\prime \prime}-\sigma(y) f^{\prime}+\left(\sigma \lambda-k^{2}\right) f=\delta(y+h), \quad(y<0) \\
f^{\prime}-\lambda f=0 \text { on } y=0 \\
f \rightarrow 0 \text { as } y \rightarrow-\infty
\end{gathered}
$$

Here, as before, the prime signifies differentiation with respect to $y$.

The formal solution of (36)-(37) can be given as follows. Let $f_{1}(y)$ and $f_{2}(y)$ be two independent solutions of the homogeneous equation of (36),

$f^{\prime \prime}-\sigma(y) f^{\prime}+\left(\sigma \lambda-k^{2}\right) f=0, \quad(y<0)$.

Then the required solution is given by the following linear combination

$$
f(y)=f_{1}\left(y_{>}\right) f_{2}\left(y_{<}\right) / W(-h),
$$

where the symbols $y_{>}$and $y_{<}$stand for

$$
\begin{aligned}
& y_{>}=y, \quad y_{<}=-h, \quad \text { for } y>-h, \\
& y_{>}=-h, \quad y_{<}=y, \text { for } y<-h,
\end{aligned}
$$

and $W(y)$ is the Wronskian

$$
W(y)=f_{1}^{\prime}(y) f_{2}(y)-f_{1}(y) f_{2}^{\prime}(y) .
$$

This solution has a unit jump in $f^{\prime}$ across $y=-h$, as required by (36). It also satisfies condition (37a) and $(37 \mathrm{~b})$ if we choose

$$
\begin{gathered}
f_{1}^{\prime}-\lambda f_{1}=0 \text { at } y=0, \\
f_{2} \rightarrow 0 \text { as } y \rightarrow-\infty .
\end{gathered}
$$

Thus, the problem is reduced to the determination of $f_{1}$ and $f_{2}$; this will be carried out in the following specific cases. Finally, the function $G(x, y)$ is given by the Fourier integral representation (35).

\section{THE PARTICULAR CASE OF CONSTANT $\sigma(y)$ IN DEEP OCEAN}

Let us consider the special case of a deep ocean with the simple property

$$
\sigma(y)=-\rho_{0}^{\prime}(y) / \rho_{0}(y)=1
$$


so that the equilibrium density increases exponentially with depth,

$$
\rho_{0}(y)=\rho_{0}(0) e^{-y} \quad(y<0)
$$

or $\rho_{0}(y)=\rho_{0}(0) \exp (-y / L)$ after the physical dimension of $y$ is restored. Although this model is somewhat artificial, it may be expected that some of the salient features of the stratification effects will be retained in this model when the ocean depth is large compared with the scaling length $L$, which is here chosen to be the rate of density growth with depth. As will be seen later, new features appear in the flow with density stratification when the motion is generated by an interior disturbance. Not only will the internal waves occur in the neighborhood of the disturbance, but the amplitude of the longrange surface waves will also be affected by the density variation. These results are shown in the present section.

Since the coefficients in the governing equation (36) are now constant, the exact solution is readily obtained. It can be shown that the following $f_{1}$ and $f_{2}$ are two linearly independent solutions of the homogeneous equation (38), satisfying conditions (40a, b), $f_{1}(y)=\left(\lambda-m_{1}\right) \exp \left(m_{2} y\right)-\left(\lambda-m_{2}\right) \exp \left(m_{1} y\right)$,

$$
f_{2}(y)=\exp \left(m_{2} y\right)
$$

where

$$
\left.\begin{array}{l}
m_{1} \\
m_{2}
\end{array}\right\}=\frac{1}{2} \pm i\left(\beta^{2}-k^{2}\right)^{\frac{1}{2}}, \quad \beta^{2}=\lambda-\frac{1}{4} .
$$

Hence from $(39 \mathrm{c})$,

$$
W(y)=-2 i\left(\beta^{2}-k^{2}\right)^{\frac{1}{2}}\left(\lambda-m_{2}\right) \exp y .
$$

Here the function $\left(\beta^{2}-k^{2}\right)^{\frac{1}{2}}$ will be defined in the complex $k$ plane with the branch cut along two vertical lines $(\beta, \beta+i \infty)$ and $(-\beta,-\beta-i \infty)$ so that

$$
\left(\beta^{2}-k^{2}\right)^{\frac{1}{2}} \rightarrow i|k| \text { as }|k| \rightarrow \infty
$$

along the real $k$ axis. Upon substitution in (35) and (39), we obtain

$$
\begin{gathered}
G(x, y)=\frac{1}{2 \pi} \operatorname{Re} \int_{-\infty}^{\infty} \exp (i k x) \frac{f_{1}\left(y_{>}, k\right) f_{2}\left(y_{<}, k\right)}{W(-h, k)} d k \\
=\operatorname{Re} \frac{1}{4 \pi i} \exp \left[\frac{1}{2}(y+h)\right] \int_{-\infty}^{\infty} \exp (i k x)\left\{\exp \left[i\left(\beta^{2}-k^{2}\right)^{\frac{1}{2}}|y+h|\right]-\exp \left[i\left(\beta^{2}-k^{2}\right)^{\frac{1}{2}}(h-y)\right]\right\} \frac{d k}{\left(\beta^{2}-k^{2}\right)^{\frac{1}{2}}} \\
\quad+\operatorname{Re} \frac{1}{2 \pi i} \exp \left[\frac{1}{2}(y+h)\right] \int_{-\infty}^{\infty} \frac{\exp \left[i k x+i\left(\beta^{2}-k^{2}\right)^{\frac{1}{2}}(h-y)\right]}{\left(\beta^{2}-k^{2}\right)^{\frac{1}{2}}-i\left(\lambda-\frac{1}{2}\right)} d k
\end{gathered}
$$

The first integral on the right-hand side of (44) is a known representation of the Hankel function of the first kind:

$\frac{1}{\pi} \int_{-\infty}^{\infty} \frac{\exp \left[i k x+i\left(\beta^{2}-k^{2}\right)^{\frac{1}{2}} y\right]}{\left(\beta^{2}-k^{2}\right)^{\frac{1}{2}}} d k=H_{0}^{(1)}(\beta r)$

with $r^{2}=x^{2}+y^{2}$. Leaving the less interesting case of $\beta^{2}<0$ till later, one may apply the identity

$$
H_{0}^{(1)}(z)=J_{0}(z)+i Y_{0}(z),
$$

to rewrite (44) as

$$
\begin{aligned}
& G(x, y)=\frac{1}{4} \exp \left[\frac{1}{2}(y+h)\right]\left[Y_{0}(\beta R)-Y_{0}\left(\beta R_{*}\right)\right] \\
& \quad+\exp \left[\frac{1}{2}(y+h)\right] \operatorname{Re} \frac{1}{2 \pi i} \\
& \quad \cdot \int_{-\infty}^{\infty} \frac{\exp \left[i k x+i\left(\beta^{2}-k^{2}\right)^{\frac{1}{3}}(h-y)\right]}{\left(\beta^{2}-k^{2}\right)^{\frac{1}{2}}-i\left(\lambda-\frac{1}{2}\right)} d k,
\end{aligned}
$$

where $R^{2}=x^{2}+(y+h)^{2}$ and $R_{*}^{2}=x^{2}+(y-h)^{2}$.

To evaluate the integral in (46), we first observe that the integrand possesses poles at the zeros of the equation

$$
i\left(\beta^{2}-k^{2}\right)^{\frac{3}{2}}=\frac{1}{2}-\lambda .
$$

By the definition of $\beta^{2}$ [see Eq. (42c)] it is readily seen that (47) has two simple zeros at $k= \pm \lambda$ for the two Riemann sheets corresponding to the two branches of the function $\left(\beta^{2}-k^{2}\right)^{\frac{1}{2}}$. According to our definition of the branch corresponding to the physical plane [see Eq. (43)], the left-hand side of (47) is purely real only for $k$ real and for $|k|>\beta$, and is there equal to $-\left(k^{2}-\beta^{2}\right)^{\frac{1}{2}}$ on our branch sheet. Therefore, real roots of (47) exist on the physical branch only when the right-hand side of (47) is negative, or

$$
\lambda>\frac{1}{2} \text {. }
$$

When $\lambda=\frac{1}{2}$ the roots at $k= \pm \beta$ reduce to two branch points, and are no longer simple poles of the integrand. 
The appearance of simple poles on the path of integration, originally taken to be along the real $k$ axis, makes the integral in (46) indeterminate as it stands. However, this indeterminacy may be removed, ${ }^{10}$ as often done in water-wave problems, by deforming the path around the poles in such a way as to satisfy the radiation condition (23). As is shown below, this can be accomplished by deforming the path to a contour $C$ circumventing both poles at $k=\lambda,-\lambda$ from below along two small semicircles, $S_{+}$and $S_{-}$, respectively, as shown in Fig. 2 (a). This deformation of the contour is determined from the behavior of the integral as $|x| \rightarrow \infty$, which is now studied. Denoting

$$
M(k, y)=\frac{\exp \left[i\left(\beta^{2}-k^{2}\right)^{\frac{1}{2}}(h-y)\right]}{\left(\beta^{2}-k^{2}\right)^{\frac{3}{2}}-i\left(\lambda-\frac{1}{2}\right)},
$$

we have

$$
\begin{aligned}
& G(x, y)-\frac{1}{4} \exp \left[\frac{1}{2}(y+h)\right]\left[Y_{0}(\beta R)-Y_{0}\left(\beta R_{*}\right)\right]=\exp \left[\frac{1}{2}(y+h)\right] \operatorname{Re} \frac{1}{2 \pi i} \int_{C} \exp (i k x) M(k, y) d k \\
= & \exp \left[\frac{1}{2}(y+h)\right] \operatorname{Re}\left\{\frac{\rho}{2 \pi i} \int_{-\infty}^{\infty} \exp (i k|x|) M(k, y) d k+\frac{1}{2 \pi i}(\underbrace{}_{S_{+}}+\underbrace{}_{S_{-}}) \exp (i k x) M(k, y) d k\right\} \\
= & \exp \left[\frac{1}{2}(y+h)\right] \operatorname{Re}\left\{\frac{1}{2 \pi i}\left[\left(\int_{C^{*}}+\int_{S_{+}}+\int_{S_{-}}\right) \exp (i k|x|) M d k+(\int_{S_{+}}+\underbrace{\int_{S^{-}}}_{S_{-}}) \exp (i k x) M d k\right]\right\} \\
= & \exp \left[\frac{1}{2}(y+h)\right] \operatorname{Re} \frac{1}{2 \pi i} \int_{C^{*}} \exp (i k|x|) M d k+H\left(\lambda-\frac{1}{2}\right) H(x)\left(2-\frac{1}{\lambda}\right) \exp [(1-\lambda) h+\lambda y] \sin \lambda x,
\end{aligned}
$$

where in $(50 \mathrm{~b}), \mathcal{O}$ denotes the Cauchy principal value and in $(50 \mathrm{c})$, the contour $C_{*}$ is the mirror image of $C$ into the real $k$ axis, as shown in Fig. 2(b), and in (50d), $H(x)$ denotes the Heaviside step function: $H(x)=1$ for $x>0$, and $H(x)=0$ for $x<0$. The procedure involved in the above operations is now explained step by step with reference to Figs. 2(a) and 2(b). First, (50b) follows at once from the construction of the contour $C$; in the first integral with the Cauchy principal value, $x$ is replaced by $|x|$ since the function $M(k, y)$ is even in $k$. Next, in (50c), the broken path of the principal-valued integral is then considered as the sum of a continuous contour $C^{*}$ and two small semicircles $S_{+}^{*}$ and $S_{-}^{*}$ in the upper half $k$ plane centered at $k=\lambda$ and $-\lambda$, respectively [Fig. 2(b)]. For $x>0, S_{+}$, and $S_{+}^{*}$ together form a closed circuit along which the resulting integral can be evaluated by the residue theorem; the same is true for the pair $S_{-}$and $S_{-}^{*}$. When $x<0$, the contributions from these semicircles cancel by pairs: $S_{+}$with $S_{-}^{*}$ and $S_{-}$with $S_{+}^{*}$. Hence (50d) results.

As $|x| \rightarrow \infty$, the Weber functions in (50) vanish, so does the integral along $C^{*}$ [see Eq. (50d)] as can be seen by applying the Riemann-Lebesgue lemma to the straight portion of the contour $C^{*}$, and by noting that the contributions from the semicircles $S_{+}^{*}$ and $S_{-}^{*}$ diminish with the exponential function $\exp (i k|x|)$ in

10 This indeterminacy disappears if the problem is solved as an initial value problem, as has been discussed by $\mathrm{J}$. J. Stoker, Water Waves (Interscience Publishers, Inc,, New York, 1957), Sec. 6.7 . the integrand as $|x| \rightarrow \infty$ since $S_{+}^{*}$ and $S_{*}^{*}$ are in the upper half $k$ plane. Now the last term in (50d) represents a train of simple waves which exist only on the downstream side $(x>0)$, the radiation condition (23) is thus fulfilled and the choice of contour $C$ is therefore correct.

The asymptotic behavior of $G(x, y)$ for large $|x|$ can be obtained as follows. First, from the known asymptotic expansion of $Y_{0}(z),{ }^{11}$ we deduce that for $|y+h|$ and $(h-y)$ both small compared with $|x|$,

$$
\begin{aligned}
& Y_{0}(\beta R)-Y_{0}\left(\beta R^{*}\right) \\
& \quad \cong \frac{2 y h}{|x|}\left(\frac{2 \beta}{\pi|x|}\right)^{\frac{1}{2}} \sin \left(\beta|x|-\frac{3 \pi}{4}\right)\left[1+O\left(\frac{1}{\beta|x|}\right)\right] .
\end{aligned}
$$

The integral along $C^{*}$ in (50d) for large $|x|$ and for $|y+h| \ll|x|$ can be evaluated for $\lambda>\frac{1}{2}$ as follows. For $x$ positive, the contour $C^{*}$ can be deformed to one around the branch cut $(\beta, \beta+i \infty)$ [see Fig. 2(b)]. Noting that arg $\left(\beta^{2}-k^{2}\right)^{\frac{1}{2}}$ is $3 \pi / 4$ (or $-\pi / 4$ ) as $k$ approaches $\beta$ on the right (or left) side of the branch cut, the integral along $C^{*}$ is reduced to the Laplace type to which Watson's lemma (see Ref. 10, p. 236) is directly applicable for large $x$. The final result for large positive $x$ reads:

$$
\begin{aligned}
& \int_{C^{*}} \exp (i k x) M d k=(2 \pi \beta)^{\frac{1}{2}} \frac{1-(h-y)\left(\lambda-\frac{1}{2}\right)}{\left(\lambda-\frac{1}{2}\right)^{2}|x|^{\frac{3}{2}}} \\
& \cdot \exp \left[i\left(\beta|x|-\frac{3 \pi}{4}\right)\right] .
\end{aligned}
$$

${ }^{11}$ G. N: Watson, Theory of Bessel Functions (Cambridge University Press, London, 1948), p. 199. 
For $x$ negative and large, the integral along $C$ in (50a) yields the same result as the last expression. Hence

$$
\begin{aligned}
& G(x, y) \cong H\left(\lambda-\frac{1}{2}\right) H(x)\left(2-\frac{1}{\lambda}\right) \\
& \cdot \exp [(1-\lambda) h+\lambda y] \sin \lambda x-\left(\frac{\beta}{2 \pi}\right)^{\frac{1}{2}}|x|^{-\frac{1}{2}} \\
& \cdot \exp \left[\frac{1}{2}(h+y)\right]\left[\frac{(h-y)\left(\lambda-\frac{1}{2}\right)-1}{\left(\lambda-\frac{1}{2}\right)^{2}}-y h\right] \\
& \cdot \sin \left(\beta|x|-\frac{3 \pi}{4}\right),
\end{aligned}
$$

from which $v(x, y)$ and other flow quantities can be calculated. This result shows that for $\lambda>\frac{1}{2}, G$ at large $|x|$ consists essentially of two modes of wave motions. One is a monochromatic wave train propagating on the downstream side; this component possesses a velocity potential and has its wavelength unaffected by density variation (physical wavelength $=2 \pi U^{2} / g$, the same as in the corresponding homogeneous flow). The other component may be interpreted as a train of internal waves, with wavelength, in physical units, given by

$$
\text { wavelength }=\frac{2 \pi L}{\beta}=2 \pi\left|\frac{\rho_{0}}{\rho_{0}^{\prime}}\right|\left(\frac{g}{U^{2}}\left|\frac{\rho_{0}}{\rho_{0}^{\prime}}\right|-\frac{1}{4}\right)^{-\frac{1}{2}}
$$

which shows the effect of density stratification. The internal wave in this case is characterized by the feature that it is attenuated, with the amplitude falling off like $|x|^{-3 / 2}$, symmetric on both sides of the disturbance. Therefore at great distances, the potential wave is the only conspicuous component left.

In addition to the above result for the range $\lambda>\frac{1}{2}$, let us consider the following cases:

$$
\lambda=\frac{1}{2}, \quad \beta=\frac{1}{2} .
$$

In this special case, the last integral in (44) also reduces to a Hankel function so that by $(45 \mathrm{a}, \mathrm{b})$ we obtain

$$
G(x, y)=\frac{1}{4} \exp \left[\frac{1}{2}(h+y)\right]\left[Y_{0}\left(\frac{1}{2} R\right)+Y_{0}\left(\frac{1}{2} R^{*}\right)\right] .
$$

Hence by (34), the velocity component $v$ is given by (with $\sigma=1$ )

$$
\begin{aligned}
v= & \frac{Q}{L} \exp \left[\frac{1}{2}(h+y)\right]\left\{Y_{0}\left(\frac{1}{2} R\right)+Y_{0}\left(\frac{1}{2} R^{*}\right)\right. \\
& \left.-\left[\frac{y+h}{R} Y_{1}\left(\frac{1}{2} R\right)+\frac{y-h}{R^{*}} Y_{1}\left(\frac{1}{2} R^{*}\right)\right]\right\} .
\end{aligned}
$$

For finite $y$ and large $|x|$ the asymptotic formula for Weber functions can be used to give

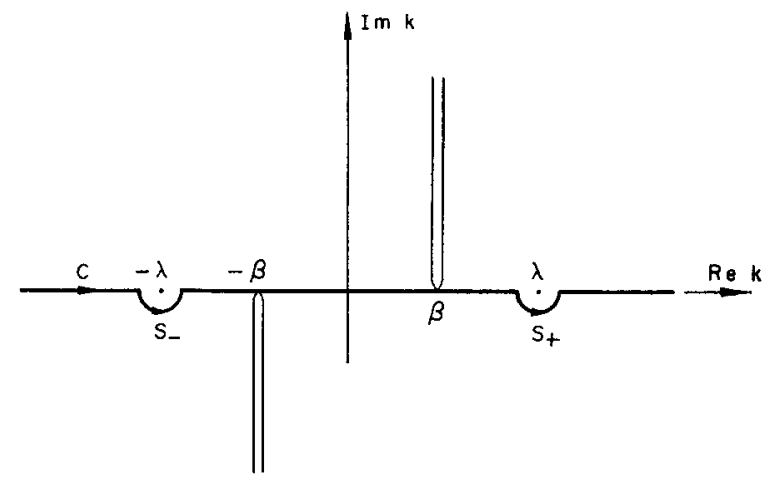

(a)

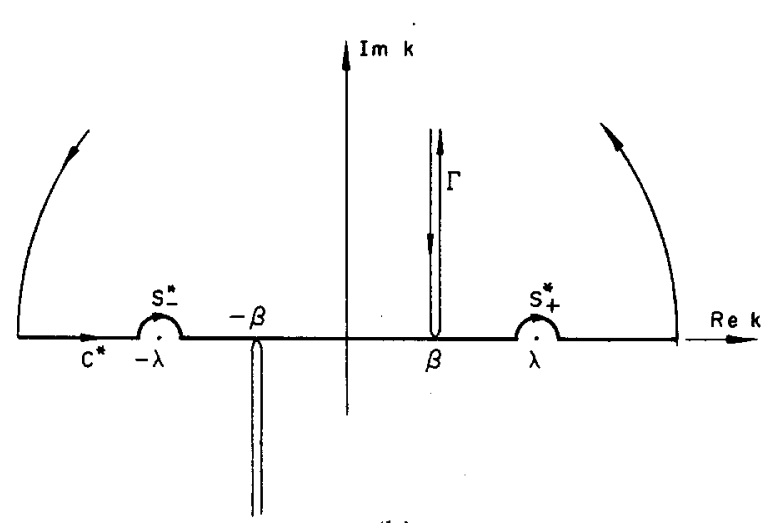

(b)

FIG. 2. The integration contours in the $k$ plane.

$v \cong \frac{Q}{2 L}(\pi|x|)^{-\frac{1}{2}} \exp \left[\frac{1}{2}(h-y)\right] \sin \left(\frac{1}{2}|x|-\frac{1}{4} \pi\right)$.

Thus, the train of simple waves ceases to exist in this special case, leaving the internal wave in the flow, now with its amplitude diminishing as $|x|^{-\frac{3}{2}}$, symmetrically in both up- and down-stream directions.

$$
\lambda<\frac{1}{4}, \quad\left(\beta^{2}<0\right) .
$$

Since $\beta$ is purely imaginary in this case, we write $\beta=i b$. Hence, by relating the Hankel function with the modified Bessel function of the second kind,

$$
i H_{0}^{(1)}(i b R)=\frac{2}{\pi} K_{0}(k R)
$$

we obtain from $(46)$

$$
\begin{aligned}
G= & -\frac{1}{2} \exp \frac{1}{2}(h+y)\left[K_{0}(b R)-K_{0}\left(b R^{*}\right)\right] \\
- & \exp \left[\frac{1}{2}(h+y)\right] \operatorname{Re} \frac{1}{2 \pi i} \\
& \cdot \int_{-\infty}^{\infty} \frac{\exp \left[i k x-\left(k^{2}+b^{2}\right)^{\frac{1}{2}}(h-y)\right]}{\left(k^{2}+b^{2}\right)^{\frac{1}{2}}+\frac{1}{2}-\lambda} d k .
\end{aligned}
$$

Both the modified Bessel functions and the above integral decay monotonically with increasing $|x|$. 
Thus we see that in this case no wave-like disturbance exists anywhere in the flow and the region of influence of the point-singularity is severely localized.

We have now obtained the following general picture. When $\lambda<\frac{1}{4}$, the disturbance caused by the point singularity is important only in a neighborhood of the singularity for there is no wave being radiated out from the disturbance. When $\frac{1}{4}<\lambda<\frac{1}{2}$, it can be shown that a train of internal waves appears symmetrically both in front and behind the disturbance, dying out at the rate $|x|^{-3 / 2}$. Though only the case of $\lambda=\frac{1}{2}$ is worked out, it is conceivable that the internal wave dies out at a slower rate proportional to $|x|^{-\frac{1}{2}}$ for $\lambda \approx \frac{1}{2}$. When $\lambda>\frac{1}{2}$ monochromatic surface waves appear which propagate to the downstream infinity while the train of internal waves decays like $|x|^{-3 / 2}$.

The fact that the simple waves exist only for $\lambda>\frac{1}{2}$ draws an analogy to the channel flow of a homogeneous fluid of finite depth. We recall that when a steady disturbance is introduced in a uniform channel flow, steady gravity waves will propagate downstream only if

$$
U^{2}<g D
$$

where $D$ is the uniform channel depth and $(g D)^{\frac{1}{2}}$ is called the critical speed. In supercritical flows no stationary gravity wave can exist. In the present case there exists an equivalent critical speed $\left(g D_{e}\right)^{\frac{1}{2}}$ since the criterion for the appearance of the longrange waves is, by (48),

$$
U^{2}<g D_{\mathrm{e}} \text { where } D_{\mathrm{e}}=-2 \rho_{0} / \rho_{0}^{\prime}=2 L
$$

in which the physical unit of $y$ is restored for clarity. The quantity $D_{\mathrm{e}}=(2 L)$ is called the equivalent channel depth due to density increase with depth.

In the limiting case of $\rho_{0}^{\prime} \rightarrow 0$, the equivalent channel depth $D_{\mathrm{e}}$ and the characteristic length $L$ tend to infinity. By adopting a new length scale $L^{*}$ (such as the depth of submergence) which is independent of $L$, for the similarity consideration, and by taking the limit $L^{*} / L \rightarrow 0$, and also $\lambda \rightarrow \infty$, we obtain the known result for a point singularity in a uniform flow of a homogeneous fluid.

$$
\begin{aligned}
\frac{v}{(Q / L)}= & \frac{1}{2 \pi}\left[\frac{y+h}{R^{2}}+\frac{y-h}{R^{*^{2}}}\right] \\
& +\frac{\nu}{\pi} \odot \int_{0}^{\infty} \frac{\cos k x}{k-\nu} \exp [k(y-h)] d k \\
& +H(x) \nu \sin \nu x \exp [\nu(y-h)],
\end{aligned}
$$

where $\nu=U^{2} / g L^{*}$, and $x, y, h$ in (58) are referred to $L^{*}$.
The long-range surface wave deserves further attention since it is responsible for the wave drag on the point singularity (or a submerged obstacle) in steady motion. To calculate the wave drag we note that for $\lambda>\frac{1}{2}$ the velocity $v$ at large $|x|$ is essentially, by (34) and (51),

$v \cong-\frac{Q}{L} H(x)(2 \lambda-1) \exp [(1-\lambda) h+\lambda y] \sin \lambda x$

Since $v$ in this region turns out to be a harmonic function, the velocity component $u$ can be written down immediately as its conjugate function.

In discussing the wave-drag problem perhaps it is more significant to take a doublet as a typical example since a circular cylinder with a radius small compared with $L$ can be represented by a doublet as the first approximation. Consider a doublet of strength $\mu$, submerged at $(0,-h)$, then (9) becomes

$$
\begin{aligned}
u_{x}+v_{y} & =\lim _{\substack{\Delta x \rightarrow 0 \\
\boldsymbol{a} \rightarrow \infty}} Q[\delta(x)-\delta(x+\Delta x)] \delta(y+h) \\
& =-\mu \delta^{\prime}(x) \delta(y+h)
\end{aligned}
$$

where $\mu$ is the limit of $(Q \Delta x)$. Hence the velocity field far behind the doublet can be obtained from (58) by differentiation with respect to $x$ and replacing $Q$ by $-\mu / L$. Thus, for $x \gg 1, y<0$,

$$
\begin{aligned}
& (u, \nu) \cong \frac{\mu}{L^{2}} \lambda(2 \lambda-1) \\
& \quad \cdot \exp [(1-\lambda) h+\lambda y](-\sin \lambda x, \cos \lambda x)
\end{aligned}
$$

The kinetic energy of the wave field per unit length of the free surface is therefore, in dimensional terms ( $x, y$, and $h$ still being dimensionless),

$$
\begin{aligned}
E_{\mathrm{k}} & =\frac{1}{2} \int_{-\infty}^{0} \rho_{0}(y)\left(u^{2}+v^{2}\right) L d y \\
& =\frac{1}{2} \rho_{0}(0) \frac{\mu^{2}}{\bar{L}^{3}} \lambda^{2}(2 \lambda-1) \exp [2(1-\lambda) h] .
\end{aligned}
$$

Hence the total energy per unit length of the free surface is ${ }^{12}$

$$
\begin{aligned}
E & =E_{\mathrm{k}}+E_{\mathrm{p}}=2 E_{\mathrm{k}} \\
& =\rho_{0}(0) \frac{\mu^{2}}{L^{3}} \lambda^{2}(2 \lambda-1) \exp [2(1-\lambda) h] .
\end{aligned}
$$

Based on the argument that the wave energy is transmitted with the group velocity, which is half the phase velocity, or $\frac{1}{2} U$, for the present case of gravity waves (59), one deduces from the energy

12 It is shown in Ref. 5 that the theorem of energy equipartition holds in general for waves in a stratified flow. 


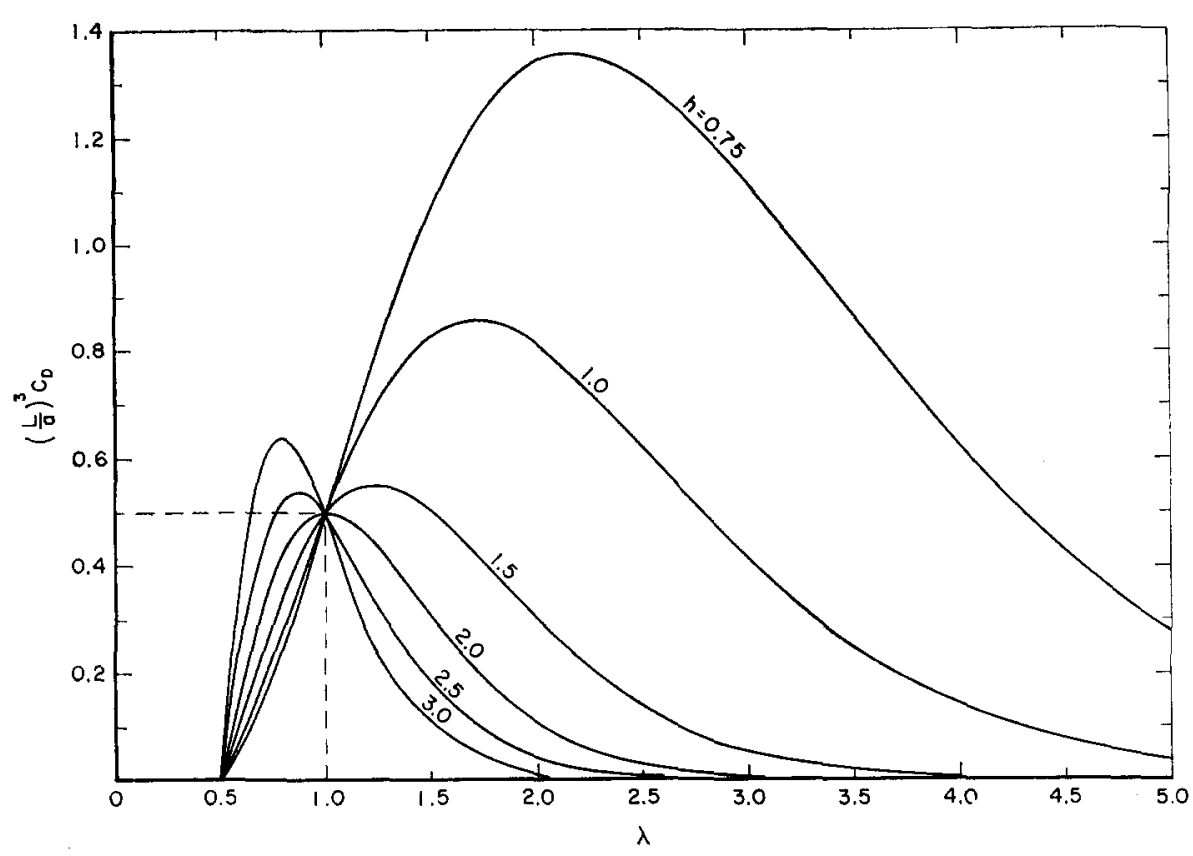

FIg. 3. Variation of the wave resistance with the internal Froude number $\lambda$ and the depth of submergence $h$. principle (see, e.g., Ref. 1, p. 415) that the wave resistance is given by

$$
X=(1 / U)\left(U-\frac{1}{2} U\right) E=\frac{1}{2} E .
$$

Finally, we obtain the wave-drag coefficient as

$$
\begin{aligned}
C_{\mathrm{D}} & =X /\left[\rho_{0}(0) \mu^{2} a^{-3}\right] \\
& =(a / L)^{3} \lambda^{2}\left(\lambda-\frac{1}{2}\right) \exp [2(1-\lambda) h] .
\end{aligned}
$$

Suppose a circular of radius $a(a \ll L)$ is approximated by a doublet, then $\mu=U a^{2}$, the above $C_{\mathrm{D}}$ is thus based on the length $a$. For $\lambda<\frac{1}{2}$, the drag is of course zero. Equation (61) is plotted in Fig. 3.

Equation (61) is an interesting result which shows the effects of the density stratification and the depth of the submergence on the wave resistance. For fixed $a / L, C_{\mathrm{D}}$ has in the region $\lambda>\frac{1}{2}$ a maximum at $\lambda=\lambda_{1}$ where

$$
\lambda_{1}=\frac{1}{4 h}\left\{(h+3)+\left[(h-1)^{2}+8\right]^{\frac{1}{2}}\right\},
$$

or

$$
h_{1}=\frac{3 \lambda_{1}-1}{\lambda_{1}\left(2 \lambda_{1}-1\right)}
$$

from which we see that $\frac{1}{2}<\lambda_{1}<1$ for $h>2$ and $\lambda_{1}>1$ for $h<2$. For each given $h$, the maximum drag is $C_{\mathbf{D} m}=(a / L)^{3} f_{m}$, with

$$
f_{m}\left(\lambda_{1}, h\right)=\lambda_{1}^{2}\left(\lambda_{1}-\frac{1}{2}\right) \exp \left[2\left(1-\lambda_{1}\right) h\right] .
$$

The minimum of $f_{m}$ for all possible $h$ is given by

$$
\frac{d f_{m}}{d h}=\left(\frac{\partial f_{m}}{\partial h}\right)_{\lambda_{1}}+\left(\frac{\partial f_{m}}{\partial \lambda_{1}}\right) \frac{d \lambda_{1}}{d h}=\frac{\partial f_{m}}{d h}=0
$$

or at

$$
\lambda_{1}=1, \quad h=2, \quad f_{m}(1,2)=\frac{1}{2} .
$$

This result shows that if the depth of submergence is $h=2$ (or $h=2 \rho_{0} / \rho_{0}^{\prime}$ in physical units), then the maximum drag coefficients experienced by the obstacle in the whole subcritical range $U<\left(\frac{1}{2} g L\right)^{\frac{1}{2}}$ has the smallest upper bound. This mini-max problem therefore indicates an optimum choice of depth of submergence for the subcritical range. Better still, we have noted that $C_{\mathrm{D}}$ vanishes in the equivalent supercritical regime $U>\left(\frac{1}{2} g L\right)^{\frac{1}{3}}$. It is noted that $C_{\mathrm{D}}$ is continuous at $U=\left(\frac{1}{2} g L\right)^{\frac{1}{2}}$.

Finally, the classical limit of the homogeneous flow is obtained by letting $L \rightarrow \infty, \lambda / L \rightarrow g / U^{2}$, so that (with the physical dimension of $h$ restored)

$$
C_{\mathrm{D}}=\left(\frac{g a}{U^{2}}\right)^{3} \exp \left(-2 g h / U^{2}\right)
$$

which is a known result. ${ }^{13} C_{\mathrm{D}}$ now decays exponentially with increasing $h$.

\section{ASYMPTOTIC SOLUTION FOR LARGE $\lambda$ AND ARBITRARY $\sigma(y)$}

As we have seen in the previous section, the final analysis of our problem depends on the explicit soultion of (36), which is a second-order ordinary differential equation with variable coefficients. It is in general very difficult to obtain the solution of

${ }^{13} \mathrm{~J} . \mathrm{V}$. Wehausen and E. Laitone, in Handbuch der Physik, edited by S. Flügge (Springer-Verlag, Berlin, 1960), Vol. IX, p. 576 . 


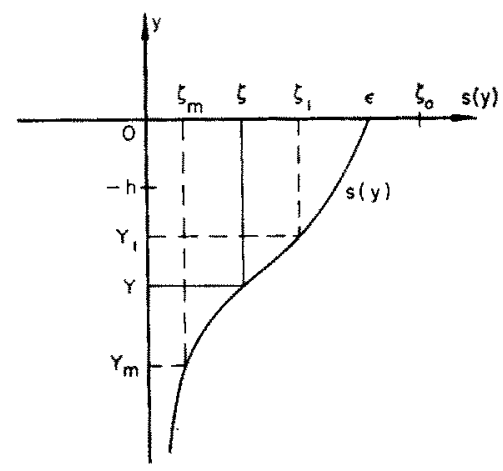

Frg. 4. The assumed stratification $\sigma(y)=\lambda s(y)$; transition point $Y$, channel depth $Y_{m}$ and wavenumber $k_{m}=\lambda \zeta_{m}{ }^{t}$

(36) in closed form except possibly for a few particular cases of $\sigma(y)$. However, for very large values of the parameter $\lambda=g L / U^{2}$, the Liouville-Langer method of asymptotic expansion ${ }^{14}$ can be adopted to investigate the asymptotic behavior of the flow field, and the result will be valid for a wide class of arbitrary $\sigma(y)$. In interpreting the large parameter $\lambda$, we may take $L$ to be the value of $\rho_{0} / \rho_{0}^{\prime}$ (with physical units restored) evaluated at $y=-h$.

In what follows we limit ourselves to large positive $\lambda$. We further assume that $\sigma(y)=-\rho_{0}^{\prime} / \rho_{0}$ is everywhere positive, decreases monotonically with increasing depth, or

$$
\sigma(y)>0, \quad \sigma^{\prime}(y)>0, \quad y \leq 0,
$$

and $\sigma(0) / \lambda$ is considered small. This assumed distribution of $\sigma(y)$, as illustrated in Fig. 4, crudely resembles a thermocline in the ocean. (The case with $\sigma(y)>0, \sigma^{\prime}(y)<0$ for $y \leq 0$ may be treated in a similar way, for which no separate account is necessary.) The condition that $\sigma^{\prime}(y) \neq 0$ for $y \leq 0$ will be seen to be a necessary condition for the present method to hold valid.

The function $f$ defined by (35) still satisfies (36). Clearly, the boundary conditions $(37 \mathrm{a}, \mathrm{b})$ remain valid, and the required solution may again be given formally by (39)-(40).

Next, we introduce the transformation

$$
f(y)=\exp [\Sigma(y)] F(y), \quad \Sigma(y)=\int_{0}^{y} \sigma(y) d y,
$$

which carries (36) to

${ }^{14} \mathrm{~A}$ description and discussion of the method has been given by A. Erdélyi [Asymptotic Expansions (Dover Publications, New York, 1956), Chap. IV]. For similar applications of Langer's method to wave propagation in inhomogeneous media, see N. A. Haskel [J. Appl. Phys. 22, 157 (1951)], B. D. Seckler and J. B. Keller [J. Acoust. Soc. Am. 31, 206 (1959)].

$$
F^{\prime \prime}+\lambda^{2}\{s(y)-\zeta+\gamma(y)\} F=0, \quad y<0, \quad y \neq h
$$

where

$$
s=\frac{\sigma(y)}{\lambda}, \quad \zeta=\frac{k^{2}}{\lambda^{2}}, \quad \gamma=\frac{\sigma^{\prime}(y)}{2 \lambda^{2}}-\frac{s^{2}}{4} .
$$

Here, $\lambda, \sigma(y)$, and $k$ may be regarded as the three principal parameters of this problem. As already mentioned before, $\lambda$ is taken positive and large, $s=\sigma / \lambda$ is assumed small and monotonically decreasing with increasing depth so that for $y \leq 0$,

$$
0 \leq s(y) \leq \epsilon, \quad 0<s^{\prime}(y)
$$

where

$$
\epsilon=s(0)=s_{0}=O\left(\lambda^{-1}\right) .
$$

Consequently, the term $\gamma(y)$ may be regarded a small quantity of higher order compared with $\epsilon$. The other parameter $\zeta$ (or $k$, the Fourier integral variable) ranges from zero to infinity, and hence no order of magnitude will be presumed for $\zeta$; we simply regard it for the moment as an independent parameter.

Now (67) is in the standard form of the classical Sturm-Liouville problem. The following two different regions of $\zeta$ are considered separately.

$$
\text { A. } \zeta>\epsilon \quad\left[\text { Or } k^{2}>\lambda \sigma_{0}, \sigma_{0} \equiv \sigma(0)\right]
$$

Although this range of $\zeta$ can also be incorporated with the following case of $\zeta<\epsilon$ by using the same general method of Langer, it is, however, simpler and more direct to consider this case separately.

By virtue of $(68)$, the factor $(\zeta-s)$ is always positive in the range $\zeta>\epsilon$. Following Liouville's method, we introduce

$\xi(y)=\int_{0}^{y}[q(y)]^{\frac{1}{3}} d y, \quad T(\xi)=[q(y)]^{\frac{1}{2}} F(y)$

with

$$
q(y)=\zeta-s(y)
$$

which transforms the interval $y \leq 0$ into $\xi \leq 0$ and (67) into

$$
\frac{d^{2} T}{d \xi^{2}}-\lambda^{2} T=\rho(\xi) T
$$

where

$$
\rho(\xi)=\frac{1}{4} q^{\prime \prime} / q^{2}-5{q^{\prime}}^{2} / 16 q^{3}-\lambda^{2} \gamma / q .
$$

Since $q^{\prime}, q^{\prime \prime}$, and $\left(\gamma \lambda^{2}\right)$ are assumed to be bounded, of order unity or less, and since $q$ is assumed to be bounded away from zero, the quantity $\rho(\xi)$ is 
bounded, and hence can be neglected as compared with the large parameter $\lambda^{z}$.

Thus, as the first approximation, (70a) becomes

$$
\frac{d^{2} T}{d \xi^{2}}-\lambda^{2} T \cong 0
$$

which has two solutions $\exp ( \pm \lambda \xi)$. As $y \rightarrow-\infty$, $\xi \rightarrow-\infty$, so for $y<-h$,

$f_{2}=\exp [\Sigma(y)] q^{-\frac{1}{2}} T_{2}=\exp [\Sigma(y)] q^{-\frac{1}{4}} \exp [\lambda \xi(y)]$.

The free surface boundary condition (37a) can be written

$f^{\prime}-\lambda f \cong \exp [\Sigma(y)]\left[F^{\prime}(y)-\lambda F(y)\right]=0, \quad y=0$

or

$$
(d T / d \xi)-\lambda q_{0}^{-\frac{1}{2}} T \cong 0, \quad \xi=0,
$$

where

$$
q_{0} \equiv q(0)=\zeta-\epsilon .
$$

All the terms neglected in (73) are of order $O\left(\lambda^{-1}\right)$ compared with those retained. Hence it is evident that for $0>y>-h$,

$$
\begin{aligned}
f_{\mathfrak{l}}=\exp [\Sigma(y)] q^{\frac{1}{2}}\left\{\left(q_{0}^{\frac{1}{2}}\right.\right. & +1) \exp (\lambda \xi) \\
& \left.+\left(q_{0}^{\frac{1}{2}}-1\right) \exp (-\lambda \xi)\right\}
\end{aligned}
$$

is a solution of (71) satisfying condition (73). The Wronskian $W$ of the above functions $f_{1}$ and $f_{2}$ is

$$
W(y, k)=-2 \lambda\left(q_{0}^{\frac{1}{2}}-1\right) \exp [2 \Sigma(y)] .
$$

Hence from (39) we obtain for $\zeta>\epsilon$

$$
\begin{aligned}
& f(y, k)=\frac{\exp \left(\frac{1}{2} \int_{-h}^{y} \sigma d y\right)}{-2 \lambda\left(q_{>} q_{<}\right)^{\frac{1}{2}}} \\
& \cdot\left\{\frac{q_{0}^{\frac{1}{2}}+1}{q_{0}^{\frac{1}{2}}-1} \exp \left[\lambda\left(\xi_{>}+\xi_{<}\right)\right]+\exp \left[\lambda\left(\xi_{>}-\xi_{<}\right)\right]\right\},
\end{aligned}
$$

where $y_{>}, y_{<}$are defined by (39b) and $\xi_{<}, q_{<}, \xi_{>}, q_{>}$, represent the functions $\xi(y)$ and $q(y)$ of (69) with $y$ assuming $y_{<}$or $y_{>}$, respectively.

In order to determine the properties of the flow field far away from the singularity we have to find first the poles of $f(y, k)$ in the complex $k$ plane, as we have done in the previous section. Since in the present interval of $\zeta>\epsilon, q_{<}$and $q_{>}$are both positive and bounded away from zero, the only poles of $f(y, k)$ are the zeros of the factor $\left(q_{0}^{\frac{1}{2}}-1\right)$, or by $(73 \mathrm{~b})$,

$$
(\zeta-\epsilon)^{\frac{1}{2}}=1 .
$$

Hence, within an error factor $[1+O(\epsilon)]$, the only poles of $f(y, k)$ are at

$$
\begin{gathered}
\zeta_{0}=1 \text { or } k_{0}= \pm \lambda . \\
\text { B. } \zeta<\epsilon \quad\left(\text { Or } k^{2}<\lambda \sigma_{0}\right)
\end{gathered}
$$

In this interval the factor $(s(y)-\zeta)$ in $(67 \mathrm{a})$ has, by virtue of the assumed monotonicity of $\sigma(y)$ [see (65)], only one simple zero, at $y=Y$, say (see Fig. 4), where

$$
s(Y)=\zeta \quad(0 \leq \zeta \leq \epsilon) .
$$

This point $Y$ is usually called a transition point, since across it the solution undergoes the transition from an oscillatory to a monotonic character.

To obtain the asymptotic solution uniformly valid in $y \leq 0$, including the transition point, we introduce, after Langer, the transformation

$$
\eta=\left(\phi^{\prime}\right)^{\frac{1}{2}} F(y)
$$

with

$$
\phi\left(\phi^{\prime}\right)^{2}=s(y)-\zeta .
$$

In terms of new variables $\eta$ and $\phi$ (67) becomes

$$
\left(d^{2} \eta / d \phi^{2}\right)+\lambda^{2} \phi \eta=\Omega \eta
$$

where

$$
\Omega=\frac{1}{2} \frac{\phi^{\prime \prime \prime}}{\left(\phi^{\prime}\right)^{3}}-\frac{3}{4} \frac{\left(\phi^{\prime \prime}\right)^{2}}{\left(\phi^{\prime}\right)^{4}}-\frac{2 \sigma^{\prime}(y)-\sigma^{2}(y)}{4\left(\phi^{\prime}\right)^{2}} .
$$

Here, as before, the primes signify differentiation with respect to $y$.

The main idea of this method may be stated as follows. Under the assumptions that $\sigma(y)$ is twice continuously differentiable for $y \leq 0$ and that $\phi^{\prime}$ is bounded away from zero, $\phi$ is a unique three times continuously differentiable real function of $y$. It then follows that $\Omega$ is a bounded function. Consequently for $\lambda$ large, the right-hand side of (82a) may be neglected as a first approximation, and the higher terms can be obtained by usual asymptotic expansions. It remains to find the condition for $\phi^{\prime}$ to be bounded away from zero.

Upon integration of (81), we obtain the (unique) real solution as

$$
\begin{gathered}
\frac{2}{3} \phi^{\frac{3}{2}}=\int_{Y}^{y}[s(y)-\zeta]^{\frac{1}{2}} d y, \\
\phi>0 \text { for } y>Y, \\
\frac{2}{3}(-\phi)^{\frac{3}{2}}=\int_{y}^{Y}[\zeta-s(y)]^{\frac{1}{2}} d y, \\
\phi<0 \text { for } y<Y,
\end{gathered}
$$


in which $Y$ is given by (79) for each fixed $\zeta$, and these square roots are interpreted by their positive branches. From this and (81) it is obvious that $\phi^{\prime}(y)$ does not vanish for $y \leq 0$, the only point not so obvious being $y=Y$. Near the transition point $y=Y$, we take the Taylor expansion of $s(y)$ in (83) and use the assumption (68), we find that

$$
\begin{array}{r}
\phi(y)=\left(s^{\prime}\right)^{\frac{1}{s}}(y-Y)\left\{1+\left(s^{\prime \prime} / 10 s^{\prime}\right)(y-Y)\right. \\
\left.+O(y-Y)^{2}\right\}
\end{array}
$$

in which $s^{\prime}$ and $s^{\prime \prime}$ are evaluated at $y=Y$. Clearly, $\phi^{\prime}(Y) \gtrless 0$ according as $s^{\prime}(Y) \gtrless 0$. It can further be shown that $\phi^{\prime}$ vanishes like $(y-Y)^{\frac{1}{3}}$ as $|y-Y| \rightarrow 0$ if $s^{\prime}(Y)=0$ and $s^{\prime \prime}(Y)=0$ (in which case the transition point is a double zero of $[s-\zeta])$. Therefore, for $\phi^{\prime}$ to be nonzero it is necessary to have $s^{\prime}(y) \neq 0$, or $\sigma^{\prime}(y) \neq 0$. This explains the assumption (65).

According to Langer's method, the asymptotic representation of $\eta$ for large $\lambda$ can be obtained by neglecting the right-hand side of $(82 a)$, or from

$$
\left(d^{2} \eta / d \phi^{2}\right)+\lambda^{2} \phi \eta=0,
$$

which has the general solution

$$
\eta=C_{1} \mathrm{Ai}\left(-\lambda^{\frac{3}{3}} \phi\right)+C_{2} \mathrm{Bi}\left(-\lambda^{\frac{2}{3}} \phi\right),
$$

where $\operatorname{Ai}(z)$ and $\operatorname{Bi}(z)$ are the Airy function of the first and second kind, and $C_{1}, C_{2}$ are arbitrary constants. The required solution $f(y)$ can be obtained from (66), (80), and (86). Since the fundamental solution $f(y)$ is required to have a unit jump across the point $y=-h$ [see Eq. (36)], we proceed to determine $f \propto f_{1}(y)$ for $y>-h$ and $f \propto f_{2}(y)$ for $y<-h$.

First, in the region $y<-h$, we note that as $y \rightarrow-\infty, \phi$ is negative and large. Hence from the known asymptotic behavior of the Airy functions ${ }^{15}$ we must set the coefficient of Bi equal to zero, giving $f_{2} \cong \exp [\Sigma(y)]\left(\phi^{\prime}\right)^{-\frac{1}{2}} \mathrm{Ai}\left(-\lambda^{\frac{2}{3}} \phi\right), \quad y<-h$.

In the region $-h<y<0$, both $\mathrm{Ai}$ and $\mathrm{Bi}$ functions may be admitted. Now, the free surface condition (37a) may be written, by (66) and (80), as

$$
\begin{aligned}
f^{\prime}-\lambda f= & \exp [\Sigma(y)]\left\{F^{\prime}-[\lambda-\sigma / 2] F\right\} \\
= & \exp [\Sigma(y)]\left(\phi^{\prime}\right)^{-\frac{1}{1}}\left[\frac{d \eta}{d y}-\lambda \eta\right. \\
& \left.+\frac{1}{2}\left(\sigma-\frac{\phi^{\prime \prime}}{\phi}\right) \eta\right]=0, \quad y=0 .
\end{aligned}
$$

To the same degree of approximation, the term $\left[\sigma-\left(\phi^{\prime \prime} / \phi^{\prime}\right)\right]$ in the above equation may be neglected

15 The important properties of the Airy functions are summarized in J. C. P. Miller, The Airy Integral, British Association Mathematical. Tables (Cambridge University Press, London, 1946). compared with $\lambda$ since this term is seen to be always bounded, and of order unity. Thus the free surface conditions simplifies to

$$
(d \eta / d y)-\lambda \eta=0, \quad y=0
$$

Application of this condition to (86) yields the asymptotic representation of $f_{1}$ as

$$
\begin{array}{r}
f_{1} \cong \exp [\Sigma(y)]\left(\phi^{\prime}\right)^{-\frac{1}{2}}\left[Q \mathrm{Ai}\left(-\lambda^{\left.\frac{2}{3} \phi\right)}-P \mathrm{Bi}\left(-\lambda^{\frac{2}{2} \phi}\right)\right],\right. \\
0>y>-h,
\end{array}
$$

where

$$
\left\{\begin{array}{l}
P \\
Q
\end{array}\right\}=\left(\frac{\partial}{\partial y}-\lambda\right)\left[\begin{array}{l}
\mathrm{Ai}\left(-\lambda^{\frac{3}{3}} \phi\right) \\
\mathrm{Bi}\left(-\lambda^{\frac{3}{3}} \phi\right)
\end{array}\right\}_{y=0}
$$

Now the Wronskian defined by (39c) can be calculated from (87), and (89), giving

$$
W(y, k) \cong \frac{1}{\pi} \lambda^{\frac{3}{3}} \exp [2 \Sigma(y)] P .
$$

Where in the last step only the leading term is retained. Finally, substituting (87)-(90) in (39a), we obtain

$$
\begin{aligned}
& f(y, k) \cong \pi \lambda^{-\frac{2}{3}} \exp [\Sigma(y)-\Sigma(-h)] \\
& . \frac{\operatorname{Ai}\left(-Z_{<}\right)\left[Q \operatorname{Ai}\left(-Z_{>}\right)-P \mathrm{Bi}\left(-Z_{>}\right)\right]}{\left[\phi^{\prime}\left(y_{>}\right) \phi^{\prime}\left(y_{<}\right)\right]^{\frac{1}{2} P}}
\end{aligned}
$$

where $y_{>}, y_{<}$are defined in $(39 \mathrm{~b})$ and

$$
Z_{>}=\lambda^{\frac{2}{3}} \phi\left(y_{>}\right), \quad Z_{<}=\lambda^{\frac{3}{3}} \phi\left(y_{<}\right) .
$$

Next, we consider the poles of the above $f(y, k)$ in the complex $k$ plane. Since $\phi^{\prime}$ is assumed nonzero, the only poles of $f(y, k)$ are the zeros of $P$, or the roots of

$P=-\lambda^{\frac{2}{3}} \phi^{\prime}(0) \mathrm{Ai}^{\prime}\left[-\lambda^{\frac{2}{3}} \phi(0)\right]-\lambda \mathrm{Ai}\left[-\lambda^{\frac{3}{2}} \phi(0)\right]=0$.

By using (81) the above equation may be written $\left(\epsilon-\zeta_{m}\right)^{\frac{1}{2}} \mathrm{Ai}^{\prime}\left(-\alpha_{m}\right)+\alpha_{m}^{\frac{1}{2}} \mathrm{Ai}\left(-\alpha_{m}\right)=0$,

$$
m=1,2,3 \cdots \text {, }
$$

where $\alpha_{m}$ and $\zeta_{m}$ are related by the following transcendental equations

$$
\begin{gathered}
\alpha_{m}=\lambda^{\frac{2}{3}} \phi_{m}(0) \equiv \lambda^{\frac{2}{3}} \phi_{0, m}, \\
\frac{2}{3} \phi_{0, m}^{\frac{3}{2}}=\int_{Y_{m}}^{0}\left[s(y)-\zeta_{m}\right]^{\frac{1}{3}} d y, \\
\zeta_{m}=s\left(Y_{m}\right) .
\end{gathered}
$$

It may be noted that for the solutions of $(92)$ to have physical significance, we must have $\zeta_{m}$ real positive, $Y_{m}$ real negative, so that both $\phi_{0, m}$ and $\alpha_{m}$ are positive real. (The negative real root $\alpha_{m}$ of (92a) is 
further discussed later.) The roots of this equation are numbered $m=1,2, \cdots$, with increasing values of $\alpha_{m}$, which correspond, by (92d), to decreasing $\zeta_{m}$.

In evaluating the zeros $\alpha_{m}$ it is worth noting the order of magnitude of various quantities involved in (92). First, (92d) implies that $\zeta_{m}$ is of order $\epsilon$ in view of (68) (see also Fig. 4). For $\zeta_{m}>0, Y_{m}$ is finite, hence the integral in (92c) is bounded. From this it follows that $\phi_{0, m}$ is about of order $O\left(\epsilon^{\frac{5}{3}}\right)$. Further inspection indicates that the roots $\alpha_{m}$ of (92a) are moderate or large in magnitude. Consequently, we may use the asymptotic expansion of $\mathrm{Ai}(z)$ for large $z$ in (92a), giving

$$
\left(\epsilon-\zeta_{m}\right)^{\frac{1}{2}} \cos \left(\theta_{m}-\frac{3 \pi}{4}\right)+\cos \left(\theta_{m}-\frac{\pi}{4}\right)=0,
$$

or

$$
\theta_{m} \equiv \frac{2}{3} \alpha_{m}^{\frac{3}{3}}=\frac{2}{3} \lambda \phi_{0, m}^{\frac{3}{3}}=\left(m-\frac{1}{4}\right) \pi+O\left(\epsilon^{\frac{1}{2}}\right) .
$$

The accuracy of these roots $\theta_{m}$ improves with increasing $m$. The solution of $\zeta_{m}$, and hence $k_{m}$, can then be deduced from (92c), (92d) and (93).

It has been noted that by virtue of the monotonicity of $\sigma(y), \zeta_{m}$ decreases whereas both $\left|Y_{m}\right|$ and $\phi_{0, m}$ increase with increasing $m$. Hence, if $\sigma^{\frac{1}{2}}(y)$ is integrable for $y \leq 0$, i.e.

$$
\int_{-\infty}^{0}(\sigma)^{\frac{1}{2}} d y \leq A
$$

$A$ being a constant, then there exists an upper limit $M$ for the integer $m$ beyond which no positive real roots of $\zeta_{m}$ will exist. In fact, from (92c) we see that $M$ must be the largest integer such that

$$
{ }_{3}^{2} \phi_{0, M}^{\frac{3}{3}} \leq \int_{-\infty}^{0}(\sigma / \lambda)^{\frac{2}{2}} d y .
$$

Using (93), we have

$$
M \leq \frac{\lambda^{\frac{1}{3}}}{\pi} \int_{-\infty}^{0}(\sigma)^{\frac{1}{2}} d y+\frac{1}{4} .
$$

In this case there exists a smallest positive real root $\zeta_{M}$ of (92), which is bounded away from zero. Thus the simple poles of $f(y, k)$ in (91) on the real $k$ axis are finite in number. Just like the previous case of constant $\sigma$, each of these simple poles $k_{m}$ will be seen to contribute a mode of wave motion. The above estimate, (95), for $M$ indicates that for fixed $\sigma(y)$, the larger the parameter $\lambda$ (or the smaller $U$ ), the more will be the excited modes. The free stream speed $U_{M}=\left(g L / \lambda_{M}\right)^{\frac{3}{2}}$ up to which the first $M$ modes are excited may be called the $M$ th critical speed.

On the other hand, if the integral of $\sigma^{\frac{3}{2}}$ is infinite, then $\phi_{m}$ increases beyond all bounds, and $k=0$ be- comes the limiting point of the sequence $\left\{k_{m}\right\}$ as $m \rightarrow \infty$. In this case there are indeed an infinite number of excited modes, regardless of the value of $\lambda$ (provided it is sufficiently large).

As a remark here, the simple pole $\zeta_{0}=1$ obtained in the previous section [see (78)] can also be calculated by the present method. To do this, we determine $\phi(y)$ anew by integrating (81) for the case of $\zeta>s(0)$, giving

$$
\frac{2}{3}\left[(-\phi)^{\frac{3}{2}}-\left(-\phi_{0}\right)^{\frac{3}{2}}\right]=\int_{y}^{0}[\zeta-s(y)]^{\frac{1}{2}} d y,
$$

in which the integration constant $\phi_{0}=\phi(0)$ is taken to be negative [see (83b)]. This constant $\phi_{0}$ may be regarded as a consequence of continuing the flow, and also $\sigma(y)$, analytically into the space $y>0$ so that the transition point $Y$ is somewhere above $y=0$, as the existence of such a transition point is inherent in the present method. Then the arguments of the Airy functions in (91) are all positive and large; use of the asymptotic expansions of $\mathrm{Ai}$, $\mathrm{Bi}$ and their derivatives ${ }^{15}$ reduces (91) to (76), the constant $\phi_{0}$ defined above being canceled out in the final result.

Having determined the poles of the function $f(y, k)$ (given by (76) for $|k|>\lambda \sigma_{0}$ and by (91) for $|k|<\lambda \sigma_{0}$ ) on the real $k$ axis, we may evaluate the integral in (35) by the residue theorem to obtain the eigen modes of the wave motion contained in the fundamental solution $G(x, y)$. Following the same argument previously used to rule out the waves propagating towards the upstream infinity, we may deform the path of integration, originally along the real $k$ axis, to the contour $C$ by circumventing along small semicircles below the poles at $k= \pm k_{m}$, $m=0,1,2, \cdots$; (see Fig. 5) where $k_{0}=\lambda$, and $k_{m}=\lambda\left(\zeta_{m}\right)^{\frac{1}{2}}, m=1,2, \cdots$, can be obtained from (92) and (93). With the contour so indented, it can readily be shown that the radiation condition is satisfied. Next, we may construct a closed contour

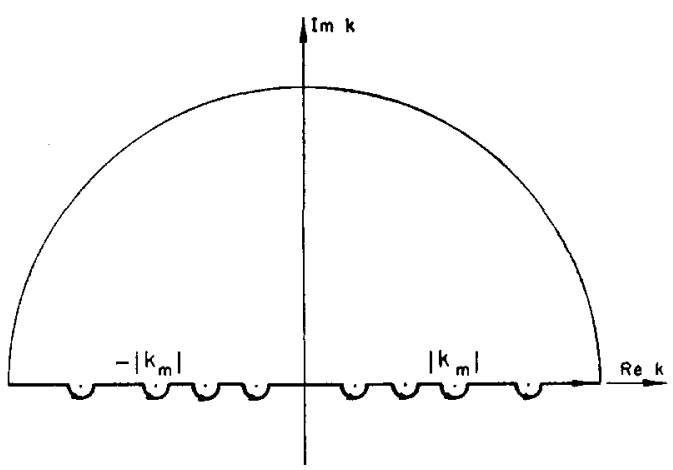

FIG. 5. The integration contour in the $k$ plane. 
by introducing a large semicircle in the upper (or lower) half $k$ plane for $x>0$ (or $x<0$ ). Finally we obtain by applying the theorem of residue that for large positive $x$,

$$
G(x, y) \sim G_{S}(x, y)+\sum_{m=1} G_{C m}(x, y)
$$

with

$$
\begin{array}{r}
G_{S}(x, y)=2 \exp [\lambda(y-h)] \sin \lambda x, \\
G_{C m}(x, y)=-2 \pi \lambda^{-\frac{2}{3}} \exp \left(\int_{-h}^{y} \frac{\sigma}{2} d y\right) \\
\frac{\mathrm{Ai}\left(-Z_{m>}\right) \mathrm{Ai}\left(-Z_{m<}\right)}{\left[\phi_{m}^{\prime}\left(y_{>}\right) \phi_{m}^{\prime}\left(y_{<}\right)\right]^{\frac{1}{2}}} \frac{Q_{m}}{(d P / d k)_{k_{m}}} \sin k_{m} x
\end{array}
$$

where $Q_{m}$ denotes $Q$ evaluated at $\phi=\phi_{m}(0)$ [see $(92 \mathrm{c})]$, and

$$
Z_{m>}=\lambda^{\frac{2}{3}} \phi_{m}\left(y_{>}\right), \quad Z_{m<}=\lambda^{\frac{2}{3}} \phi_{m}\left(y_{<}\right) .
$$

The notations $\phi_{m}\left(y_{>}\right)$and $\phi_{m}\left(y_{<}\right)$are self-evident; they assume the form of (83) except with $Y_{m}$ replacing $Y$ and $y_{>}$or $y_{<}$taking the place of $y$.

The factor $Q_{m} /(d P / d k) k_{m}$ in $(96 \mathrm{c})$ can be further evaluated as follows. From (89b), (81), (93) and the asymptotic expansions of $\mathrm{Bi}$ and $\mathrm{Bi}^{\prime}$, we find

$$
\begin{aligned}
Q_{m} & =\left\{\left(\frac{\partial}{\partial y}-\lambda\right) \mathrm{Bi}\left[-\lambda^{\frac{2}{3}} \phi_{m}(y)\right]\right\}_{y=0} \\
& =-\lambda^{\frac{3}{3}} \phi_{0, m}^{\prime} \mathrm{Bi}^{\prime}\left(-\alpha_{m}\right)-\lambda \mathrm{Bi}\left(-\alpha_{m}\right) \\
& \sim-(\pi)^{-\frac{1}{2}}\left[\lambda^{\frac{2}{3}} \phi_{0, m}^{\prime} \alpha_{m}^{\frac{1}{4}} \cos \left(\theta_{m}-\frac{\pi}{4}\right)\right. \\
& \left.+\lambda \alpha^{-\frac{1}{3}} \cos \left(\theta_{m}+\frac{\pi}{4}\right)\right] \sim(-)^{m+1} \lambda \pi^{-\frac{1}{2}} \alpha_{m}^{-\frac{1}{4}}
\end{aligned}
$$

Similarly, we have

$$
\begin{aligned}
\left(\frac{d P}{d k}\right)_{k_{m}} & =\frac{d}{d k_{m}}\left[\left(\frac{\partial}{\partial y}-\lambda\right) \mathrm{Ai}\left(-\lambda^{\frac{3}{3}} \phi_{m}\right)\right]_{y=0} \\
& \sim-\lambda \frac{d}{d k_{m}} \operatorname{Ai}\left(-\alpha_{m}\right) \\
& =\lambda \mathrm{Ai}^{\prime}\left(-\alpha_{m}\right) \frac{d \alpha_{m}}{d k_{m}} \\
& \sim(-)^{m+1} \pi^{-\frac{1}{2}} k_{m} \alpha_{m}^{-\frac{1}{4}} \int_{Y_{m}}^{0}\left[s(y)-\zeta_{m}\right]^{-\frac{1}{2}} d y .
\end{aligned}
$$

Therefore

$$
\frac{1}{Q_{m}}\left(\frac{d P}{d k}\right)_{k_{m}} \sim \frac{k_{m}}{\lambda} \int_{Y_{m}}^{0}\left[s(y)-\zeta_{m}\right]^{-\frac{1}{2}} d y .
$$

\section{Discussion of the Result}

The fundamental solution $G$ of (96) consists of two parts. One component $G_{S}$ is a harmonic function of $(x, y)$, and hence possesses a velocity potential; it is identical to the surface wave of the corresponding flow of a homogeneous fluid at the same value of $\lambda$. That this mode arises in the flow is to be expected since $e^{\lambda y} \sin \lambda x$ is a simple solution of the homogeneous problem, regardless of the nature of $\sigma(y)$, insofar as the stratification is stable. This component is called the surface wave mode. A comparison between $(96 \mathrm{a})$ and $(50 \mathrm{~d})$ shows that the surface mode terms in both cases become asymptotically equal for large $\lambda$.

The component $G_{C}$ has the following salient features. From the definition (83), $\phi_{m}(y) \gtrless 0$ according as $y \gtrless Y_{m}$, and evidently, $G_{C_{m}}(x, y)$ oscillates for $y>Y_{m}$ and decays exponentially for $y<Y_{m}$. From this it readily follows that the $m$ th mode, which is represented by the $m$ th term in the series of $(96 \mathrm{c})$, oscillates in $0>y>Y_{m}$ and decays exponentially for $y<Y_{m}$. Thus, $Y_{m}$ plays the role of the depth of a channel within which the $m$ th mode is effectively trapped. Furthermore, the amplitudes of the waves $G_{C_{m}}$ all vanish (at least by the approximation of the present case) at the free surface $y=0$, i.e.

$$
G_{C m}(x, 0) \cong 0 \quad m=1,2,3, \cdots .
$$

since (92) implies approximately

$$
\operatorname{Ai}\left[-z_{m>}\right]=\operatorname{Ai}\left[-\lambda^{\frac{2}{3}} \phi_{m}(0)\right] \cong 0 .
$$

For the above reason the components $G_{C m}$ is called the internal (or channeled) gravity waves. Their velocity fields are no longer irrotational.

It is important to note that the roots $\alpha_{m}$ of (92a) depend on both the stratification function $\sigma(y)$ and the parameter $\lambda$, but are independent of the submergence depth $h$. Hence $\alpha_{m}$ are all determined once $\sigma$ and $\lambda$ are given, regardless of the value of $h$. The corresponding wavenumber $k_{m}=\lambda\left(\zeta_{m}\right)^{\frac{1}{2}}$ form a decreasing sequence since $\zeta_{0}=1>\zeta_{1}>\zeta_{2}>\cdots$; hence the wavelengths, $l_{m}=2 \pi L / k_{m}$ (with the physical units restored), of the various modes form an increasing sequence

$$
l_{0}<l_{1}<l_{2}<\cdots l_{m}<\cdots .
$$

It has also been pointed out that the channel depths of the internal modes, $Y_{m}$, increase with $m$. Thus, these internal waves oscillate both in the stream direction and the vertical direction within the various channel widths, with increasing wavelength and channel width for increasing $m$. The irrotational 


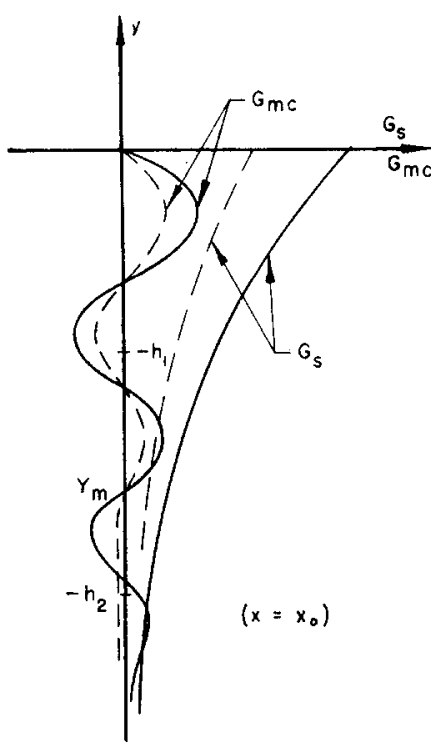

(a)
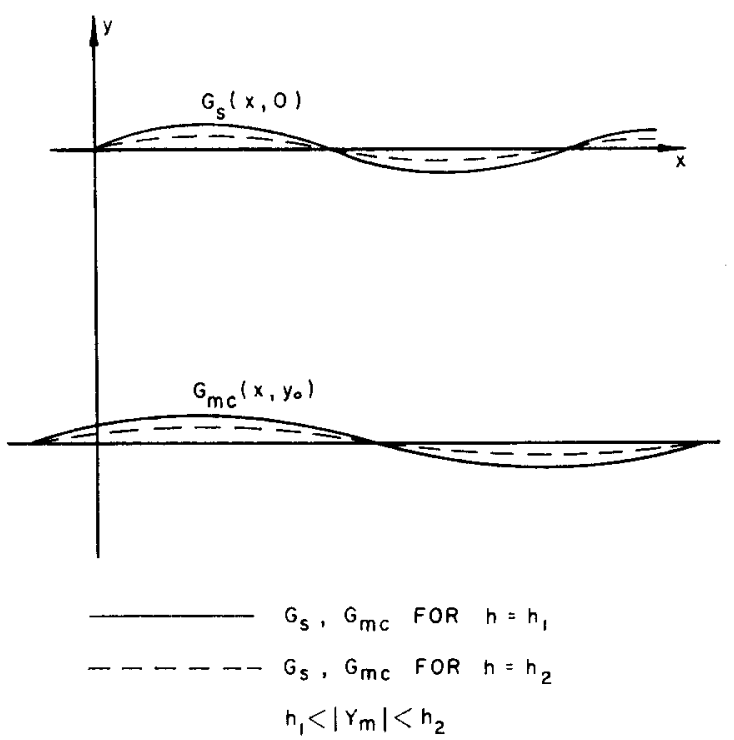

(b)
Fig. 6. Effect of depth of submergence on gravity waves $G_{s}, G_{m c}$. (a) At $x=x_{0}$, far downstream.

(b) Profile of $G_{s}, G_{m}$. surface wave has the shortest wavelength of all the gravity waves.

Another feature of interest is the effect of submergence depth $h$. First, the exponential factor in (96c) gives an exponential decay of $\left|G_{C m}\right|$ with increasing depths (decreasing $y$, for fixed $h$ ) and increasing submergence $h$ (for $y$ fixed). Furthermore, from $(83),(96 c, d)$ it is straightforward to deduce this following result (Table $\mathbf{I}$ ): in which,+- denote $>0,<0$, respectively, and "oscil." and "decay" stand for "oscillating" and "decaying" exponentially with increasing depths." From this result it is evident that the amplitude of the $m$ th mode $y$-component oscillation within the channel $\left(Y_{m}<y<0\right)$ is further damped out by an additional exponentially decay factor in $\mathrm{Ai}\left(-Z_{m<}\right)$ after the disturbance descends beneath the corresponding channel depth $Y_{m}$, leaving no oscillatory motion in $y<Y_{m}$. Therefore, when the submergence is sufficiently deep, the internal waves, of rather weak amplitude, will be virtually trapped in the channels which are at some distance above the disturbance. These salient features of the motion are illustrated in Fig. 6.

Finally, the following distinctions between the two special cases treated in this work are noteworthy. In the first case of constant $\sigma$, there is only one internal mode, and this wave motion is attenuated according to a power law at large distances. In contrast, the additional degrees of freedom associated with arbitrary stratification function $\sigma(y)$ result in more internal wave modes, there being finite $(M<\infty)$ or infinite number of these modes according as whether $\sigma^{\frac{1}{3}}(y)$ is integrable or not for $0>y>-\infty$ [see (94)]. Furthermore, these internal waves now propagate to the downstream infinity unattenuated.

Let us investigate the first few internal modes in greater detail. For the first few modes $\phi_{0, m}$ are small for large $\lambda$ [see (92b)]; and hence by $(92 \mathrm{c}), Y_{m}$ are also small. We further recall that in our model, $s^{\prime}(0)>0$; we shall confine ourselves to a class of $s(y)$ such that the following approximation is valid for the determination of the first few modes:

$$
s(y) \sim \epsilon+s^{\prime}(0) y .
$$

Hence from (92) and (93),

$$
\begin{aligned}
\frac{2}{3} \lambda \phi_{0, m}^{\frac{3}{2}} & =\lambda \int_{Y_{m}}^{0}\left[s(y)-\zeta_{m}\right]^{\frac{1}{2}} d y \cong \lambda\left[s^{\prime}(0)\right]^{\frac{1}{2} \frac{2}{3}\left(-Y_{m}\right)^{\frac{3}{2}}} \\
& =\theta_{m} \cong\left(m-\frac{1}{4}\right) \pi,
\end{aligned}
$$

from which we deduce the channel depths

$$
Y_{m} \cong-\left\{\frac{3 \pi}{2}\left(m-\frac{1}{4}\right)\left[\lambda^{2} s^{\prime}(0)\right]^{-\frac{1}{2}}\right\}^{\frac{2}{3}} \quad m=1,2, \cdots
$$

and the wavenumber

$k_{m}=\lambda\left(\zeta_{m}\right)^{\frac{1}{2}}=\lambda\left[s\left(Y_{m}\right)\right]^{\frac{1}{2}} \cong \lambda\left[\epsilon+s^{\prime}(0) Y_{m}\right]^{\frac{1}{2}}$.

TABLE I.

\begin{tabular}{clllll}
\hline \hline & & $Z_{m>}$ & $Z_{m<}$ & $\operatorname{Ai}\left(-Z_{m>}\right)$ & $\operatorname{Ai}\left(-Z_{m<}\right)$ \\
\hline (I) & $y>Y_{m}$ & + & + & oscil. & oscil. \\
$-h>Y_{m}$ & $y<Y_{m}$ & + & - & oscil. & decay \\
(II) & $y>Y_{m}$ & + & - & $\begin{array}{l}\text { oscil. } \\
\text { decay }\end{array}$ & $\begin{array}{l}\text { decay } \\
\text { decay }\end{array}$ \\
\hline \hline
\end{tabular}


Furthermore, from (97) we obtain,

$$
\frac{1}{Q_{m}}\left(\frac{d P}{d k}\right)_{k_{m}} \cong \frac{2 k_{m}}{\lambda}\left[-\frac{Y_{m}}{s^{\prime}(0)}\right]^{\frac{1}{3}} \text {. }
$$

The wavelength of the $m$ th mode, with the physical units restored, is

$$
l_{m}=\frac{2 \pi}{k_{m}} L=\frac{2 \pi U^{2}}{g}\left[\epsilon+s^{\prime}(0) Y_{m}\right]^{-\frac{1}{2}} .
$$

The above results present an approximate estimate of the physical quantities of the first few internal wave modes, whose qualitative features have already been discussed.

Another quantity of interest is the amplitude of the internal wave relative to that of the irrotational surface wave. We consider the case of a doublet as a typical example of a closed body. For a doublet of strength $\mu$ we repeat the argument leading to (59) and use (10) to obtain the perturbed stream function (with the choice of $1 / \sigma(-h)$ as the characteristic length $L$ ) as

$$
\begin{gathered}
\psi_{\text {doublet }}=\frac{\mu}{L^{2}}\left(\frac{\partial}{\partial h}-1\right) G(x, y) \\
\cong \psi_{s}+\sum_{m} \psi_{c m} \quad x \rightarrow \infty
\end{gathered}
$$

where

$$
\begin{aligned}
\psi_{S} & =-\left(\mu / L^{2}\right) 2 \lambda \exp [\lambda(y-h)] \sin \lambda x, \quad(106 \mathrm{~b}) \\
\psi_{c m} & =-\left(\frac{2 \pi \mu}{L^{2}}\right) \exp \int_{k}^{y} \frac{\sigma}{2} d y \frac{Q_{m}}{(d P / d k)_{k_{m}}}\left[\frac{\phi_{m}^{\prime}(-h)}{\phi_{m}^{\prime}(y)}\right]^{\frac{1}{2}} \\
& \left\{\left[\frac{d \mathrm{Ai}(z)}{d z}\right]_{z=-\lambda^{2 / 3} \phi_{m}(-h)}\right\} \mathrm{Ai}\left[-\lambda^{\frac{2}{3}} \phi_{m}(y)\right] \sin k_{m} x .
\end{aligned}
$$

By using the results (100)-(104) it is quite straightforward to derive from the above expressions the following order-of-magnitude estimate of $\psi_{s}$ at $y=0$ and $\psi_{c_{m}}$ at $y=-h$ (assuming $-h>Y_{m}$ for a legitimate estimate of the amplitude of $\psi_{C_{m}}$ ) as

$$
\begin{aligned}
&\left|\psi_{s}\right| \cong 2\left(\mu / L^{2}\right) \lambda \exp (-\lambda h) \\
&\left|\psi_{c_{m}}\right| \cong 2\left(\mu / L^{2}\right)\left|\frac{Q_{m}}{(d P / d k)_{k_{m}}}\right| \\
& \sim 4\left(\mu / L^{2}\right)\left[\frac{3 \pi}{2}\left(m-\frac{1}{4}\right)\right]^{\frac{1}{3}} \frac{\sigma_{0}^{\frac{1}{3}}}{\left(\sigma_{0}^{\prime}\right)^{\frac{2}{3}}} \lambda^{-\frac{1}{6}}
\end{aligned}
$$

From this result we note that only for very shallow submergences (or when the parameter $\lambda h=g h_{*} / U^{2}$, $h_{*}$ in the physical dimension, is small) does the surface mode become important as compared with the internal waves. In the limit of $\lambda h \rightarrow 0$, the ratio $\left|\psi_{s} / \psi_{c_{m}}\right|$ is of order $\lambda^{7 / 6}$. However, since the surface wave decays exponentially with increasing

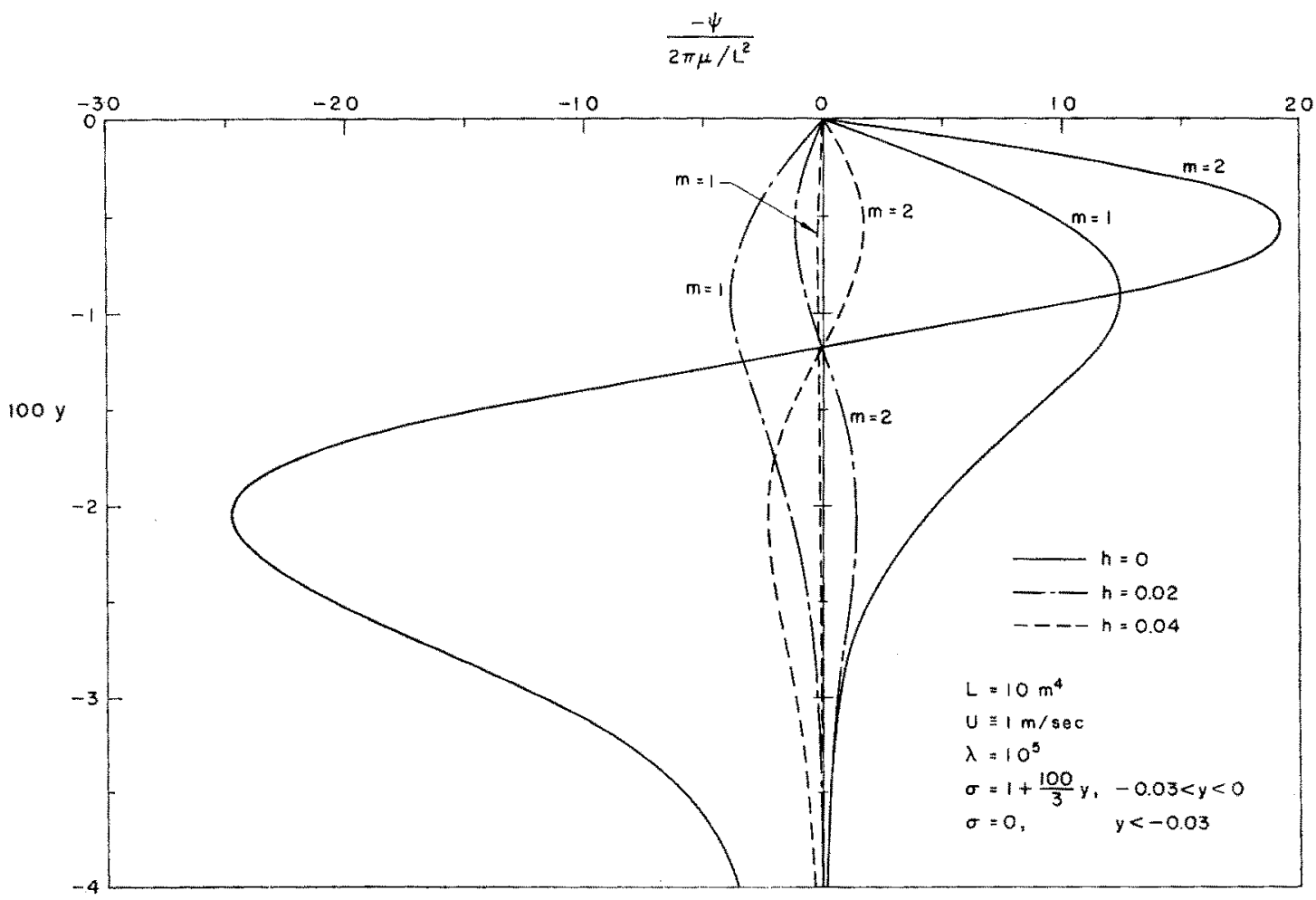

FIG. 7. Variation of the amplitude of the internal waves with $y$ for several depths of submergence. 
depth and since the internal waves reside in channels of finite widths, there may exist an appreciably wide range of $h$ whereby the internal wave modes are fully excited, whereas the surface wave becomes negligibly small.

In order to achieve a more concrete picture of the motion, we consider the following numerical example

$$
\begin{array}{r}
\rho_{0}\left(y_{*}\right)=\rho_{0}(0) \exp \left\{-\left(y_{*}+\frac{y_{*}^{2}}{600}\right) \times 10^{4}\right\}, \\
\left(0>y_{*}>-300 \mathrm{~m}\right), \\
\rho_{0}\left(y_{*}\right)=\rho_{0}(-300), \quad\left(y_{*}<-300 \mathrm{~m}\right),
\end{array}
$$

and

$U=1 \mathrm{~m} / \mathrm{sec}, \quad I=10^{4} \mathrm{~m}, \quad \lambda \cong 10^{5}$,

so that

$\sigma(y)=1+\frac{100}{3} y \quad\left(0>y \equiv y_{*} / L>-0.03\right)$,

$\sigma(y)=0, \quad(y<-0.03)$.

This simple case may be regarded as a crude approximation of the measured distribution given by Fig. 1 to the same order of magnitude. It is found that for this density stratification there exists only two internal wave modes with wavelengths $l_{1}=$ $286 \mathrm{~m}$ and $l_{2}=662 \mathrm{~m}$ and the corresponding channel depths $Y_{1^{*}}=-156 \mathrm{~m}$ and $Y_{2^{*}}=-273 \mathrm{~m}$, respectively. The variation of $\psi_{c_{m}}(y)$ is calculated from (106) for the stations where $\sin k_{m} x=1$, as shown in Fig. 7, for three different depths of submergence, $h_{*}=0 \mathrm{~m}, 200 \mathrm{~m}, 400 \mathrm{~m}$ (or $h=0$, $0.02,0.04)$. It may be noted that for each fixed $y,-\psi(x, y)$ is equal to the vertical displacement of the streamline at that depth $y$. In comparison with these internal waves, the surface mode has the value $\psi /\left(2 \mu / L^{2}\right)=10^{5}$ at $y=0$, but becomes infinitesimal for $h=0.02$ due to the exponential decay. Furthermore, we note that for a circular cylinder of radius $R$, the factor $\left(\mu / U L^{2}\right)$ is essentially $(R / L)^{2}$.

Note added in Proof: ${ }^{16}$ In a private communication Chia-Shun Yih points out to the authors that the analogy between the critical speeds of a homogeneous open channel flow and of a stratified flow, as mentioned in the discussion pertinent to Eq. (56) and again in Sec. 4 of the text, is not a complete one for the reason that the dispersion relationship for the irrotational wave mode, which may be imbedded in a spectrum of internal waves, retains the same form $\left(c^{2}=g / k\right)$ as in a homogeneous flow of infinite depth. Our explanation for this distinction is that the phenomena of sub- and super-critical flows in open channels arise solely from the dispersion relation, whereas in stratified flows they are the manifestation of the effect of density stratification on the wave amplitude. Thus the analogy is introduced in a broader sense to include both the frequency effect in open channel flows and the amplitude effect in stratified flows.

\section{ACKNOWLEDGMENT}

This work was supported by the U. S. Office of Naval Research under Contract Nonr-220(35).

\footnotetext{
${ }^{16}$ We are grateful to Professor Yih for enlightening discussions of this topic.
} 